\title{
L'intonation des énoncés interrogatifs dans la parole des apprenants finnophones du français
}

\author{
Mari Wiklund (iD) and Anne Riippa \\ Université de Helsinki \\ Email: mari.wiklund@helsinki.fi
}

(Received 07 March 2021; revised 03 September 2021; accepted 27 September 2021; first published online 9 December 2021)

\begin{abstract}
Résumé
Cette étude porte sur l'intonation des énoncés interrogatifs dans la parole des apprenants finnophones du français. Le corpus consiste en des enregistrements où 15 apprenants finnophones du français et un groupe de contrôle de 5 locuteurs natifs du français lisent un extrait d'un dialogue tiré de la pièce de théâtre En attendant Godot (Beckett, 1952). Les résultats montrent qu'aussi bien les apprenants finnophones que les locuteurs natifs du français produisent une montée intonative à la fin des questions totales d'information. En ce qui concerne les questions partielles, les résultats sont plus divergents : le plus souvent, les apprenants finnophones produisent aussi une montée intonative à la fin d'une question partielle, tandis que dans la parole des locuteurs natifs, l'intonation descendante et plate sont nettement plus fréquentes.

Comme l'intonation montante n'a pas de fonction interrogative établie en finnois, cette étude apporte des preuves supplémentaires du fait que tous les traits prosodiques de la parole des locuteurs L2 ne peuvent pas être expliqués par la transmission directe de la L1. La fréquence des contours montants dans la parole des apprenants finnophones est probablement liée au fait que l'importance de monter l'intonation à la fin des énoncés interrogatifs est soulignée dans l'enseignement du FLE.
\end{abstract}

\section{INTRODUCTION}

Lorsque l'on apprend une nouvelle langue, il ne suffit pas seulement d'apprendre à prononcer correctement les sons de la langue cible. En effet, la prononciation réussie d'une langue étrangère nécessite aussi l'apprentissage de la prosodie de la langue cible (Mennen et De Leeuw, 2014: 183), puisque chaque langue a un système intonatif qui lui est propre et qui est différent de celui des autres (Hirst et Di Cristo, 1998). Le français, par exemple, se caractérise par des mouvements intonatifs larges (e.g. Di Cristo, 1998; Mertens, 2008; Morel et Danon-Boileau, 1998; Rossi, 1999), tandis que l'intonation du finnois se caractérise par une certaine monotonie créée par une échelle de variations restreinte de la fréquence fondamentale (Iivonen, 1998). Par conséquent, la comparaison de ces deux 
langues typologiquement distantes (langue romane vs. langue finno-ougrienne) est particulièrement intéressante.

La fonction interrogative d'un contour intonatif montant a parfois été considérée comme un trait universel (Gussenhoven, 2004). En finnois, un contour montant n'a cependant pas de fonction interrogative établie (Iivonen, 1998). En français, toutes les questions ne se terminent pas par un contour montant non plus (DelaisRoussarie et al., 2015; Delais-Roussarie et Herment, 2018; Di Cristo, 1998; Santiago et Delais-Roussarie, 2015). Y a-t-il des différences à noter en ce qui concerne l'intonation des phrases interrogatives dans la parole des apprenants finnophones du français et les locuteurs natifs du français? L'objectif de notre étude est de répondre aux questions suivantes: 1) Les apprenants finnophones $\mathrm{du}$ français produisent-ils typiquement une montée intonative / une chute intonative / une intonation plate dans les mêmes contextes que les locuteurs natifs du français? 2) Y a-t-il des différences à noter en ce qui concerne les valeurs de l'étendue des mouvements intonatifs ? 3) Comment pourrait-on expliquer les différences éventuelles, et quelles sont leurs implications pour l'enseignement du français comme langue étrangère?

Notre corpus consiste en des enregistrements où 15 apprenants finnophones du français $(n=15)$ et 5 locuteurs natifs du français $(n=5)$ lisent à haute voix un extrait d'un dialogue tiré de la pièce de théâtre En attendant Godot (Beckett, 1952: 118-119). Les deux pages que les informateurs ont lues sont riches en phrases interrogatives, ce qui nous avait fait choisir cet extrait au préalable. Les locuteurs finnophones avaient lu et traduit l'extrait en classe avant les enregistrements.

Les locuteurs finnophones sont des étudiants qui ont suivi un cours de français au Centre de langues de l'Université de Helsinki (Finlande). Leur niveau de français oral est A1-A2 (CEFR, 2021). Les locuteurs natifs sont des étudiants Erasmus à l'Université de Helsinki. Le corpus sera décrit d'une manière plus détaillée dans le chapitre 2 ('Corpus et méthodes').

En ce qui concerne le choix d'un texte littéraire au détriment d'un texte ordinaire, nous avons voulu choisir un extrait littéraire issu d'une pièce de théâtre puisque le théâtre est un genre littéraire qui permet d'aborder également la langue par sa phonétique et sa prosodie, et non pas seulement à travers sa sémantique. Les pièces de théâtre sont écrites pour être lues et jouées. Ainsi sont-elles naturellement riches en "variété des intonations à prêter à un message " (Defays, Delbart, Hammami et Saenen, 2014: 51). En effet, la lecture d'une pièce de théâtre à haute voix a l'avantage de sensibiliser l'apprenant à la prosodie de la langue cible ainsi qu'à l'idée que la façon dont on parle compte autant que ce que l'on dit. Lire du théâtre permet aussi aux informateurs d'imiter de façon ludique la communication. Chaque dialogue fictif est naturellement une imitation de la communication authentique, ce qui rend la lecture ludique. Plus c'est ludique, plus c'est motivant pour l'apprenant.

Notre choix du texte littéraire ainsi que l'approche didactique s'inspirent donc des théories d'apprentissage élaborées par des chercheurs ayant travaillé sur l'emploi et les avantages de la littérature en FLE, parmi lesquels le didacticien belge Jean-Marc Defays et les didacticiens français Marie-Claude Albert et Marc Souchon. Defays et ses collègues défendent l'approche pédagogique selon laquelle " l'apprentissage d'une langue étrangère ne consiste pas seulement en une 
activité cognitive qui sollicite l'intelligence, mais engage toute la personnalité de l'apprenant : la socialisation (participation, intégration, ouverture, tolérance), le psychoaffectif (émotion, imagination, confiance en soi, curiosité, esprit ludique) le physique (prononciation, attitude et gestuelle, comportement) " (Defays, Delbart, Hammami et Saenen, 2014: 17). Étant donné que l'apprentissage d'une langue étrangère est un processus exigeant qui ne sollicite pas simplement l'intelligence mais aussi le psychoaffectif, la lecture d'un texte littéraire en classe de langues, elle, est particulièrement apte à contribuer de manière efficace à ce processus d'apprentissage, et ce, tout simplement parce que la fiction est une forme de langage particulièrement affective qui décrit et évoque des émotions.

Albert et Souchon (2000: 50) affirment à leur tour que ce sont la grande plasticité, la disponibilité et le caractère ouvert de la littérature « qui font de la communication littéraire une forme de communication privilégiée dans l'enseignement et l'apprentissage d'une langue étrangère ", même s'il faut effectivement avoir acquis un certain bagage linguistique et culturel afin de pouvoir comprendre le sens global du texte lu (Defays, Delbart, Hammami et Saenen, 2014: 8). D'après nous, il est temps de laisser derrière nous les réserves concernant les capacités des apprenants de langues étrangères à surmonter les difficultés qu'ils rencontrent à la lecture d'un texte littéraire sous prétexte que celui-ci contient des phrases et des énoncés qui sont improbables dans des conversations réelles. Dans ce type d'activité, comme dans n'importe quelle activité, les apprenants peuvent et doivent être aidés par leur enseignant, naturellement.

Lire et faire lire un texte littéraire écrit en une langue étrangère n'est pas un défi insurmontable, ni pour les apprenants ni pour les enseignants. Bien au contraire. C'est un défi qui vaut la peine d'être surmonté puisque la lecture d'une pièce de théâtre à haute voix, par deux, en classe de FLE, a l'avantage de permettre aux apprenants de se familiariser avec la culture en question, par le biais d'une activité d'interaction qui leur est bénéfique sur plusieurs niveaux, pas moins sur le plan affectif.

Comprendre le rôle des émotions dans l'apprentissage des langues étrangères est un domaine de recherches qui n'a pas encore été massivement élaboré. Mais à l'heure actuelle, nombreux sont les didacticiens qui s'intéressent à comprendre le rôle des émotions dans l'apprentissage (Beirne, 2020; Dewaele, 2005 ; Pekrun et Linnenbrink-Garcia, 2014).

\subsection{Intonation des énoncés interrogatifs en français}

D’une manière générale, il est difficile d'assigner un sens ou une fonction à un contour mélodique (Delais-Roussarie et Herment, 2018). En effet, la relation entre l'intonation et la valeur interrogative est complexe. Selon Delais-Roussarie et Herment (2018), l'intonation constitue seulement un des facteurs distinctifs entre les questions et les assertions. Selon les auteurs, les généralisations concernant ce sujet sont cependant fréquentes, et notamment les grammaires et les manuels de français langue étrangère (FLE) insistent souvent sur la réalisation de l'intonation montante à la fin des énoncés interrogatifs. Nous reviendrons sur les manuels de FLE dans le chapitre 4.

En français, deux types de questions totales peuvent être distinguées : 'questions totales de confirmation' (qui anticipent une réponse spécifique, par exemple: «Tu 
viendras bien à la fête demain ?») et 'questions totales d'information' (par exemple : «Tu veux un verre de vin ?»). Selon certaines études antérieures (Di Cristo, 1998; Fónagy et Bérard, 1973), les questions totales de confirmation se terminent typiquement par une chute intonative, précédée d'un pic intonatif porté par la syllabe pénultième. Les résultats de Delais-Roussarie et al. (2015) suggèrent cependant que les questions totales de confirmation se terminent en une intonation montante $(\mathrm{H} \%)$.

Les questions totales d'information se terminent typiquement par une montée intonative portée par la dernière syllabe accentuée de l'énoncé (Di Cristo, 1998: 204). Selon Delais-Roussarie et al. (2015), si une question totale d'information est construite sans marqueur morpho-syntaxique d'interrogation (inversion du sujet, marqueur d'interrogation) comme dans l'énoncé «Tu as faim ? ", l'intonation sert dans une grande mesure à distinguer la question d'une déclaration ou d'une exclamation. Dans ce cas, la phrase interrogative se termine typiquement par une montée intonative $(\mathrm{H} \%)$, qui peut être très haute ou pas. Si un marqueur lexical ou morpho-syntaxique d'interrogation est utilisé, la forme du contour intonatif est moins stable: la montée finale n'a pas besoin d'être très haute, et elle est précédée d'une chute portée par la syllabe accentuée (Delais-Roussarie et al., 2015).

Les questions partielles sont marquées par la présence d'un mot interrogatif ('qui', 'quand', 'pourquoi', 'comment', 'où', etc.), ce qui suffit pour indiquer la modalité interrogative d'un énoncé. On peut distinguer entre les 'questions partielles neutres' (Di Cristo, 1998: 205) - ou les 'questions partielles d'information' (Delais-Roussarie et al., 2015) - utilisées pour obtenir une nouvelle information, et les 'questions partielles échos' par lesquelles le locuteur demande d'une reformulation d'une question ou d'une réponse qu'il n'a pas comprise ou qui lui semble bizarre ou surprenante (par exemple:A : «Tu pèses combien?» B: «Je pèse combien? »)1. Selon Di Cristo (1998: 205), les questions partielles échos sont généralement caractérisées par un haut niveau intonatif et par une montée finale ressemblant à celle typique des questions totales d'information. Selon l'étude de Delais-Roussarie et al. (2015), les questions partielles échos comportent typiquement une montée intonative suivie d'une chute (comme les questions totales échos), ou bien, uniquement une montée finale. S'il s'agit de l'expression de la surprise en répétant une question ou une assertion de l'interlocuteur, la question partielle écho se termine par une montée intonative $(\mathrm{H} \%)$, et la syllabe pénultième est allongée (Delais-Roussarie et al., 2015).

Les questions partielles neutres (par exemple: «Quand est-ce que tu viens?» / "Combien d'heures as-tu travaillé hier ?»), en revanche, se terminent typiquement par une chute intonative finale (L\%), ressemblant à celle d'une assertion, et une des syllabes du mot interrogatif ou de la locution 'est-ce que' porte un pic intonatif (Delais-Roussarie et al., 2015; Di Cristo, 1998: 205). Selon Delais-Roussarie et al. (2015) les autres formes sont cependant également possibles: la chute intonative peut par exemple être suivie d'une montée intonative. La variation apparaissant à la fin des questions partielles n'a pas de fonction contrastive (Delais-Roussarie et al., 2015). Les questions construites avec la locution 'est-ce

\footnotetext{
${ }^{1}$ Évidemment, la place du mot interrogatif ainsi que la longueur de l'énoncé peuvent également avoir un impact sur le contour final dans ce type de cas.
} 
que' se terminent systématiquement en un contour clairement descendant (L\%) dans le corpus étudié par Delais-Roussarie et al. (2015). ${ }^{2}$

Selon Di Cristo (1998: 206), si le mot interrogatif apparaît à la fin de l'énoncé, le contour intonatif est comme celui typique d'une assertion : l'intonation monte jusqu'à la fin du groupe verbal et descend ensuite au moment de la production du mot interrogatif. Néanmoins, les résultats de Delais-Roussarie et al. (2015) suggèrent que si le mot interrogatif apparaît à la fin de l'énoncé, la question se termine typiquement par une montée finale $(\mathrm{H} \%)$, et elle constitue un seul groupe accentuel.

Dans les études portant sur la prosodie de la langue seconde (L2), il est souvent dit que le système prosodique de la langue maternelle (L1) influence le processus d'apprentissage et aboutit à l'interférence entre la L1 et la L2 (Mennen, 2007 ; Rasier et Hiligsmann, 2007 ; Santiago et Delais-Roussarie, 2015). Autrement dit, on considère souvent que les traits prosodiques d'un locuteur d'une $\mathrm{L}^{3}$ s'expliquent par le système prosodique de sa langue maternelle. Néanmoins, bien que plusieurs études aient montré que l'influence du système prosodique de la L1 joue effectivement un rôle important dans l'explication des formes prosodiques trouvées dans la parole d'un locuteur d'une L2, il arrive aussi que l'on trouve des formes prosodiques dans la parole L2 qui ne peuvent être identifiées ni dans la L1 ni dans la langue cible (L2) du locuteur en question (Jilka, 2007). Il est également connu que bien que certains types de questions (par exemple les questions partielles) se terminent typiquement en une chute intonative aussi bien dans la L1 que dans la L2 d'un locuteur, le locuteur a tendance à les produire avec une intonation montante dans la L2 (Horgue, $2010^{4}$; MacDonald, 2011 ; Santiago et Delais-Roussarie, 2015). Certains contours montants qui apparaissent aux premières phases du processus d'apprentissage et qui diminuent progressivement aux phases ultérieures peuvent éventuellement traduire l'incertitude des locuteurs L2 (Horgue, 2010 ${ }^{5}$ ).

Santiago et Delais-Roussarie (2015 : 245) remarquent aussi que les occurrences fréquentes des contours intonatifs montants dans les phrases interrogatives peuvent aussi être liées au fait que la fonction interrogative d'un contour intonatif montant est parfois considérée comme un trait universel (Gussenhoven, 2004). De ce fait, il s'agirait de l'activation de cette fonction universelle lorsqu'un locuteur commence à apprendre à parler une langue étrangère (Santiago et Delais-Roussarie, 2015 : 245). Néanmoins, comme déjà dit plus haut, par exemple dans la langue finnoise, le contour montant n'a pas de fonction interrogative établie (Iivonen, 1998).

Santiago et Delais-Roussarie (2015) ont étudié l'intonation des questions en espagnol et en français, ainsi que dans la parole des apprenants hispanophones $\mathrm{du}$ français. Selon leurs résultats, les questions totales d'information sont généralement produites avec un contour intonatif montant en espagnol, tandis qu'en français, une grande variété de contours pouvait être trouvée. De plus, la

\footnotetext{
${ }^{2}$ Le corpus de Delais-Roussarie et al. (2015) est très large et varié. Il comporte des données de neuf différents endroits pour les trois variétés les plus importantes du français : Öil, Occitan et Franco-Provençal.

${ }^{3}$ Nous utilisons dans cet article l'abréviation 'L2' dans un sens large pour faire référence aux apprenants d'une langue étrangère (L2, L3, L4, etc.).

${ }^{4}$ Thèse de doctorat non publiée citée par Santiago et Delais-Roussarie (2015:245).

${ }^{5}$ Thèse de doctorat non publiée citée par Santiago et Delais-Roussarie (2015: 245).
} 
segmentation en groupes accentuels est généralement marquée par un pic intonatif $\left(\mathrm{H}^{\star}\right)$ en français, tandis qu'en espagnol, les limites de groupes accentuels ne sont pas nécessairement marquées d'une manière tonale. En ce qui concerne les interrogations produites par les apprenants hispanophones du français, une très grande proportion de mouvements intonatifs extra-montants pouvait être trouvée. En revanche, les limites de groupes accentuels n'étaient pas marquées d'une manière tonale à l'intérieur des énoncés. Les auteurs constatent qu'il s'agit probablement dans ce cas d'activation de la transmission des traits prosodiques de la L1 à la L2 (Santiago et Delais-Roussarie, 2015: 267). Néanmoins, en ce qui concerne les questions partielles d'information, les auteurs remarquent (p. 267) que l'emploi fréquent des contours montants - et surtout des contours extra-montants ne peut pas être expliqué par la transmission de la L1. En effet, dans ce type de questions, les contours non-montants sont aussi fréquents que les contours montants en espagnol, tandis que les apprenants hispanophones du français emploient un contour montant dans la plupart des cas (80\%). De plus, le contour extra-montant ( $\mathrm{HH} \%)$ est nettement plus souvent utilisé par les apprenants L2 que par les locuteurs natifs, quelle que soit la langue, et les apprenants du niveau débutant (A2) emploient ce contour plus souvent que les apprenants plus avancés. Selon les auteurs (p. 267), l'emploi fréquent des mouvements extra-montants peut être lié soit 1) à l'association universelle des contours montants à la fonction interrogative, soit 2) à l'incertitude linguistique des apprenants.

\subsection{Intonation des énoncés interrogatifs en finnois}

Selon Iivonen (1998: 317), en finnois, le contour descendant concerne d'une manière générale aussi bien les assertions que les questions. Le niveau mélodique final y est le même: il est bas dans les deux cas (Iivonen, 2009: 73). La voix craquée et le non-voisement sont fréquents en finnois à la fin d'un énoncé (Iivonen, 2009). Le niveau mélodique initial des questions est généralement haut, tandis que dans les assertions, il est nettement plus bas. L'échelle de variations mélodiques est restreinte dans les assertions, tandis que dans les questions, l'échelle de variations est très large (Iivonen, 2009: 73). La forme globale des assertions est descendante. Dans les questions, l'intonation monte généralement jusqu'au dernier mot, et ensuite, elle redescend. L'accentuation est globalement faible dans les assertions; en revanche, dans les questions, le dernier mot est fortement accentué (Iivonen, 2009 : 73).

Néanmoins, les énoncés peuvent également se terminer en une montée intonative en finnois (Iivonen, 1978), et dans la parole spontanée, les formes de contours intonatifs sont d'une manière générale variées (Iivonen, Nevalainen, Aulanko et Kaskinen, 1987). Selon Iivonen (1998: 326) et Anttila (2008), on peut trouver des occurrences des contours montants surtout dans les questions-tags et dans les questions elliptiques. Un nouveau résultat apporté par l'étude d'Anttila (2009) est qu'en fait, on peut trouver des contours montants dans différents types de questions d'une manière récurrente. Anttila (2009: 176) constate que cela peut être causé par l'interférence avec d'autres langues, comme par exemple l'anglais. Il peut également s'agir d'une "nouvelle manière" de faire appel à l'interlocuteur (Anttila, 2009: 176) ou d'exprimer de la politesse (Anttila, 2008: 90). 


\section{CORPUS ET MÉTHODES}

\subsection{Corpus}

Nous avions sélectionné comme informateurs 20 étudiants universitaires qui se répartissaient en deux groupes. Le premier groupe consistait en 15 apprenants finnophones du français langue étrangère (L2). Ce qui rapprochait les étudiants du premier groupe, c'est qu'à l'époque des enregistrements, ils suivaient tous le même cours de français pour les débutants du niveau A1 (CEFR, 2021) au Centre de Langues de l'Université de Helsinki (Finlande). La langue maternelle de tous les participants de ce groupe était le finnois. ${ }^{6}$ L'âge moyen des participants de ce groupe était de 25 ans. La plupart $(17 / 20,85 \%)$ d'entre eux étaient des femmes. Sur le plan acoustique, parmi les informateurs finnophones, 12 voix féminines et 3 voix masculines ont été identifiées et analysées. (Un des informateurs n'avait pas indiqué son genre sexuel dans la fiche d'informations de base.)

Le deuxième groupe de participants était le groupe de contrôle. Celui-ci consistant en cinq étudiantes Erasmus qui étaient des locutrices natives du français, d'origine française $(3 / 5)$ ou belge (2/5). Elles faisaient leurs études à l'Université de Helsinki à l'époque, à l'automne 2018. Elles avaient été scolarisées dans différentes villes françaises (Lyon, Lacanau, Poitiers) et belges francophones (Binche, Theux). L'âge moyen des participants du groupe de contrôle était de 21 ans.

La tâche des informateurs consistait à lire à haute voix un extrait d'un dialogue tiré de la pièce de théâtre En attendant Godot (Beckett, 1952: 118-119). Le texte lu est donné en annexe (Annexe 1). La parole a été enregistrée par des professionnels de la technologie audiovisuelle dans une pièce silencieuse. Pendant l'enregistrement, chaque informateur avait un microphone très près de la bouche. Les informateurs ont porté un casque micro pour que la distance entre les lèvres et le microphone ne change pas. Les participants avaient l'occasion de se familiariser avec le texte avant de le lire à haute voix. Ils avaient également l'occasion de poser des questions sur le contenu du texte s'ils ne le comprenaient pas.

\subsection{Méthodes}

D’abord, nous avons choisi cinq énoncés interrogatifs différents dans le dialogue lu par les informateurs (Beckett, 1952: 118-119) pour être étudiés plus en détail. Les énoncés interrogatifs choisis représentent les différentes catégories de questions apparaissant dans l'extrait lu. Les énoncés interrogatifs sélectionnés sont les suivants:

- Questions totales :

1) «Passons maintenant à autre chose, veux-tu ?»

2) «Ça va mieux?»:

- Questions partielles:

3) «Qu'est-ce qu'il y a de si extraordinaire?»

4) «Pourquoi?»

5) «Qui êtes-vous?»:

\footnotetext{
${ }^{6}$ Une participante avait aussi une deuxième langue maternelle: le russe.
} 
Ensuite, nous avons effectué des analyses acoustiques à l'aide du logiciel Praat (Boersma et Weenink, 2021). Lors de ces analyses, nous avons mesuré l'étendue (en demi-tons, dt) du mouvement mélodique (mouvement de la courbe de fréquence fondamentale, $f_{0}$ ) apparaissant à la fin de chaque question. Les formes de ces mouvements ont été étudiées à l'aide de Prosogram (Mertens, 2004), un outil technique permettant de générer des annotations des extraits de la parole et des stylisations correspondant à une estimation de la hauteur mélodique perçue par un auditeur moyen. ${ }^{7}$ Plus précisément, Prosogram présente graphiquement des variations mélodiques qui dépassent le seuil de glissando (ou le seuil de perception) ou non ; autrement dit, cet outil technique décrit des variations perçues comme un changement (montée ou chute) plutôt que comme une mélodie statique. L'avantage de ce système est qu'il est indépendant de la langue, ce qui est important lorsque l'on étudie la parole des apprenants L2. Dans les analyses des formes des mouvements mélodiques, nous avons utilisé le modèle présenté par Santiago et Delais-Roussarie (2015: 249) dans leur étude portant sur les énoncés interrogatifs en français, en espagnol et dans la parole des apprenants hispanophones du français. Quatre contours différents sont distingués dans ce modèle: ${ }^{8}$

\section{1) Contour descendant (code : L\%)}

O Chute mélodique lors de laquelle la $f_{0}$ descend plus de 2 demi-tons ${ }^{9}$ entre la syllabe pénultième et la fin de la dernière syllabe du groupe intonatif. ${ }^{10} \mathrm{Le}$ contour est perçu comme un mouvement descendant.

2) Contour plat (code: $0 \%$ )

○ La courbe $f_{0}$ reste stable entre la syllabe pénultième et la dernière syllabe de groupe intonatif. Le contour est perçu comme un plateau bas.

\footnotetext{
${ }^{7}$ Nous avons choisi de présenter les formes acoustiques des échantillons de parole à l'aide de Prosogramme notamment puisque ce système était utilisé aussi dans Santiago et Delais-Roussarie (2015) dans leur étude portant sur les énoncés interrogatifs en français, en espagnol et dans la parole des apprenants hispanophones du français. Comme nous utilisons dans cette étude le modèle présenté par Santiago et Delais-Roussarie (2015: 249) dans les analyses des formes des mouvements mélodiques, il est logique d'utiliser le même outil technique que ces auteurs. De plus, Prosogramme génère une stylisation des mouvements intonatifs qui convient bien à ce type d'étude.

${ }^{8}$ Les mesures ont été prises en tenant compte du contour visible avec le logiciel Praat (Boersma et Weenink, 2021). En ce qui concerne les contours montants (H\% et HH\%), ce sont les valeurs de la $f_{0}$ minimum et $f_{0}$ maximum lors de la production de la dernière syllabe de l'énoncé qui ont été mesurées. Quant aux contours plats $(0 \%)$, la $f_{0}$ moyenne de la syllabe pénultième et la $f_{0}$ moyenne de la dernière syllabe ont été mesurées. Dans le cas des contours descendants (L\%), la $f_{0}$ maximum de la syllabe pénultième et la $f_{0}$ minimum de la dernière syllabe ont été mesurées. Les valeurs ont été extraites manuellement une par une à l'aide du logiciel Praat (Boersma et Weenink, 2021), pour que les erreurs possibles de la courbe $f_{0}$ aient pu être détectées et corrigées.

${ }^{9}$ Dans l'étude de Santiago et Delais-Roussarie (2015), l'étendue des chutes mélodiques était d'environ $2 \mathrm{dt}$. Dans nos données, l'étendue typique des chutes mélodiques est nettement supérieure à $2 \mathrm{dt}$. Par conséquent, nous avons défini la chute (L\%) comme un mouvement descendant dont l'étendue est supérieure à $2 \mathrm{dt}$.

${ }^{10}$ Un 'groupe intonatif correspond à l'unité la plus large dans la segmentation intonative. Dans le système F_ToBi (Delais-Roussarie et al., 2015), cette unité est appelée 'Intonational Phrase'. Un groupe intonatif se caractérise par la présence d'un ton de frontière à sa frontière droite et d'un fort allongement de la syllabe finale. Il est souvent suivi d'une pause. Si plusieurs propositions se succèdent dans la parole, celles-ci forment typiquement chacune un groupe intonatif indépendant. Dans notre corpus, chaque énoncé interrogatif forme un groupe intonatif indépendant, puisqu'il s'agit d'un texte lu.
} 
3) Contour montant (code: H\%)

O Mouvement montant qui commence à l'attaque de la dernière syllabe d'un groupe intonatif. L'étendue du mouvement est au maximum $10 \mathrm{dt}$. Le contour est perçu comme un mouvement montant.

4) Mouvement extra-montant (code: $\mathrm{HH} \%)$

O Mouvement extra-montant qui commence à l'attaque de la dernière syllabe du groupe intonatif et continue jusqu'au plafond de la tessiture du locuteur. L'étendue du mouvement est de plus de $10 \mathrm{dt}$. Le contour est perçu comme un mouvement très fortement montant.

Après avoir mesuré les valeurs de l'étendue et analysé les formes des mouvements mélodiques apparaissant à la fin des questions choisies, nous avons comparé les résultats des deux groupes d'informateurs et analysé chaque énoncé interrogatif plus en détail. ${ }^{11}$ Le débit (syllabes/sec) ainsi que l'écart-type de la $f_{0}(\mathrm{dt})$ et la moyenne de la $f_{0}(\mathrm{~Hz})$ lors de la production de chaque énoncé interrogatif ont également été mesurés afin de décrire la forme acoustique globale de l'énoncé. Pour finir, les moyennes et les écarts-types des valeurs de l'étendue des contours montants des deux groupes d'informateurs ont été calculés (test de ShapiroWilk) et comparés (test-t de Welch).

\section{RÉSULTATS}

Nous commençons la présentation des résultats de cette étude par les questions totales (3.1). Ensuite, nous passons aux questions partielles (3.2). Pour finir (3.3), nous présentons les résultats quantitatifs concernant les valeurs de l'étendue des montées intonatives apparaissant à la fin des questions totales et des questions partielles. ${ }^{12}$

\subsection{Questions totales}

Selon nos résultats, aussi bien les apprenants finnophones du français que les locuteurs natifs du français produisent un contour montant $(\mathrm{H} \%)$ ou un contour extra-montant $(\mathrm{HH} \%)$ à la fin des questions totales d'information. Cela est conforme aux études antérieures selon lesquelles les questions totales d'information du français se terminent typiquement en une montée intonative portée par la dernière syllabe accentuée de l'énoncé (Delais-Roussarie et al., 2015; Di Cristo, 1998: 204). Delais-Roussarie et al. (2015) remarquent aussi que si un marqueur lexical ou morpho-syntaxique d'interrogation est utilisé, la forme du contour intonatif est moins stable, et la montée finale n'a pas besoin d'être très haute. En effet, dans l'étude de Santiago et Delais-Roussarie (2015), une grande variété de contours pouvait être trouvée à la fin des questions totales d'information en français. En ce qui concerne la parole des apprenants

\footnotetext{
${ }^{11}$ Les occurrences des tons initiaux seraient intéressantes à étudier aussi, puisque les tons initiaux peuvent avoir une influence sur le choix des tons finals. Dans cette étude, nous avons cependant décidé de nous concentrer uniquement sur les tons finals.

${ }^{12}$ Tous les 20 informateurs ont été analysés pour obtenir les résultats quantitatifs présentés dans ce chapitre. Dans les exemples donnés, la parole de différents locuteurs finnophones et francophones natifs est présentée sous forme de courbes et valeurs acoustiques.
} 
hispanophones du français, une très grande proportion de mouvements intonatifs extra-montants pouvait être trouvée, ce qui peut s'expliquer par le fait qu'en espagnol, ce type de questions sont généralement produites avec un contour intonatif montant (Santiago et Delais-Roussarie, 2015).

Dans notre corpus, dans le cas de l'énoncé interrogatif «Passons maintenant à autre chose, veux-tu ?», construit avec l'inversion du sujet, aussi bien les locuteurs natifs que les apprenants L2 produisent typiquement une montée intonative ( $\mathrm{H} \%)$ dont l'étendue moyenne est de 5,9 dt pour les locuteurs natifs et de 5,5 dt pour les apprenants L2. Un locuteur finnophone produit une chute intonative (L\%), et un autre termine la question par une intonation plate (0\%). Tous les locuteurs natifs produisent un contour montant $(\mathrm{H} \%)$ à la fin de cette question.

En ce qui concerne l'énoncé interrogatif "Ça va mieux?", construit sans marqueur lexical ou morpho-syntaxique d'interrogation, l'emploi du contour extramontant (HH\%) est plus fréquent: $6 / 15$ apprenants L2 (40\%) et 3/5 des locuteurs natifs $(60 \%)$ terminent cette question par un contour extra-montant $(\mathrm{HH} \%)$. Pour les apprenants L2, l'emploi du contour montant $(\mathrm{H} \%)$ est cependant encore plus fréquent $(8 / 15,53 \%)$ que l'emploi du contour extra-montant (HH\%). Néanmoins, aussi bien les locuteurs natifs que les apprenants L2 terminent typiquement cette question en une montée intonative ( $\mathrm{H} \%$ ou $\mathrm{HH} \%)$ : seulement un apprenant finnophone produit une chute intonative (L\%) à la fin de cette question, et l'intonation plate $(0 \%)$ n'y apparait pas du tout. Les nombres d'occurrences de différents tons de frontière ainsi que les valeurs de l'étendue moyennes des mouvements mélodiques concernant les questions totales sont donnés dans le tableau 1. La colonne présentant les valeurs des apprenants finnophones $(n=15)$ est indiquée avec l'abréviation 'FIN', et la colonne qui donne les valeurs des locuteurs natifs $(n=5)$ est indiquée avec l'abréviation 'FR'.13

\subsubsection{Exemple 1: "Passons maintenant à autre chose, veux-tu ?»}

Les interrogations totales portent sur la phrase entière sans avoir recours aux mots interrogatifs. Il est, en principe, possible d'y répondre par «oui» ou "non». Très souvent, pour simplifier la présentation des phrases interrogatives dans l'enseignement du FLE, les exemples illustrant les interrogations totales sont des phrases simples. Néanmoins, comme nous le savons, une interrogation totale peut se juxtaposer, dans une phrase complexe, avec une proposition affirmative ou exclamative tout en constituant une phrase grammaticalement correcte. Selon Coveney (2011: 12), les interrogations totales s'expriment généralement uniquement par l'intonation qui est le plus souvent - mais pas systématiquement - montante. Dans le premier exemple, la modalité interrogative est cependant également indiquée par l'inversion du sujet clitique («veux-tu»). Cet énoncé est particulièrement intéressant puisqu'il comporte, en fait, deux modalités: interrogative et impérative.

La figure 1 présente la forme acoustique de la question totale «Passons maintenant à autre chose, veux-tu ?", produite par une apprenante finnophone

\footnotetext{
${ }^{13}$ Trois tableaux présentant les valeurs de l'étendue des mouvements mélodiques finals de toutes les questions étudiées produites par tous les informateurs du corpus sont donnés en annexe (Annexe 2). Ces tableaux comportent aussi les moyennes et les écarts-types de la $f_{0}$ de chaque locuteur.
} 
Tableau 1. Questions totales: types du ton de frontière et valeurs de l'étendue moyennes des mouvements mélodiques finals. Apprenants finnophones (FIN) et locuteurs natifs (FR)

\begin{tabular}{|c|c|c|c|c|}
\hline Question & $\begin{array}{l}\text { Type du ton de } \\
\text { frontière (FIN), } \\
\text { nombre } \\
\text { d'occurrences }\end{array}$ & $\begin{array}{l}\text { Étendue moyenne } \\
\text { du mouvement } \\
\text { mélodique final } \\
\text { (dt) (FIN) }\end{array}$ & $\begin{array}{l}\text { Type du ton } \\
\text { de frontière } \\
\text { (FR), nombre } \\
\text { d'occurrences }\end{array}$ & $\begin{array}{l}\text { Étendue moyenne du } \\
\text { mouvement } \\
\text { mélodique final (dt) } \\
\text { (FR) }\end{array}$ \\
\hline $\begin{array}{l}\text { «Passons main- } \\
\text { tenant à autre } \\
\text { chose, veux-tu?» }\end{array}$ & $\begin{array}{l}\mathrm{H} \%: 13 \\
\mathrm{~L} \%: 1 \\
0 \%: 1\end{array}$ & $\begin{array}{l}\mathrm{H} \%:+5,5 \mathrm{dt} \\
\mathrm{L} \%:-17.0 \mathrm{dt} \\
0 \%:-1,7 \mathrm{dt}\end{array}$ & $\mathrm{H} \%: 5$ & $\mathrm{H} \%:+5,9 \mathrm{dt}$ \\
\hline «Ça va mieux?» & $\begin{array}{l}\text { H\%: } 8 \\
\text { L\%:1 } \\
\text { HH\%: } 6\end{array}$ & $\begin{array}{l}\mathrm{H} \%:+6,8 \mathrm{dt} \\
\mathrm{L} \%:-2.2 \mathrm{dt} \\
\mathrm{HH} \%:+12,5 \mathrm{dt}\end{array}$ & $\begin{array}{l}\mathrm{H} \%: 2 \\
\mathrm{HH} \%: 3\end{array}$ & $\begin{array}{l}\mathrm{H} \%:+8,8 \mathrm{dt} \\
\mathrm{HH} \%:+11,6 \mathrm{dt}\end{array}$ \\
\hline
\end{tabular}

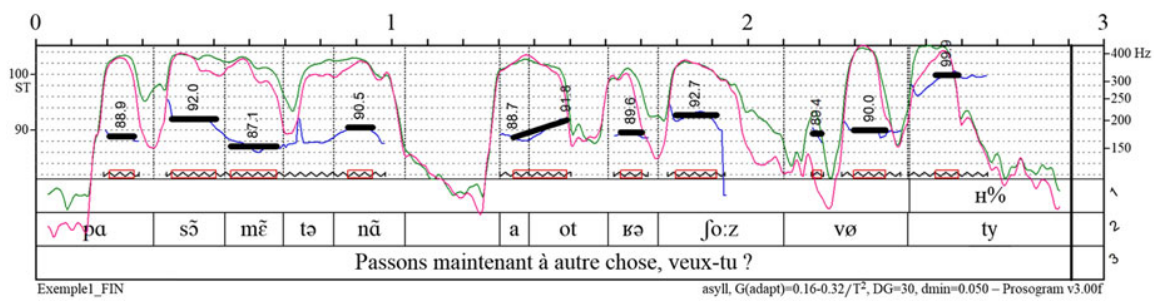

Figure 1. Question totale «Passons maintenant à autre chose, veux-tu ?» produite par une apprenante finnophone du français.

du français. ${ }^{14}$ Les courbes acoustiques ont été générées avec Prosogram (Mertens, 2004). Le trait épais dans la figure donne une estimation de la hauteur perçue par un auditeur moyen. Les traits fins correspondent aux différents paramètres acoustiques. Le trait bleu donne la fréquence fondamentale. Le trait vert donne l'intensité, et le trait rouge indique le signal sonore filtré. La ligne en zigzag indique les parties voisées. Comme on peut le voir dans la figure 1, l'informatrice produit un contour montant $(\mathrm{H} \%)$ à la fin de la question. L'étendue de la montée (mesurée de l'attaque de la syllabe accentuée jusqu'à la fin de cette syllabe) est de 4,1 dt.

La figure 2, quant à elle, donne la forme acoustique de la même question produite par une locutrice native du français. Dans sa parole aussi, l'intonation monte à la fin, lors de la production de la dernière syllabe accentuée ([ty]). L'étendue de la montée (H\%) est de 4,5 dt. En ce qui concerne le mouvement intonatif final, il n'y a donc pas de grandes différences à noter entre les deux locutrices dans ce cas. La locutrice finnophone parle un peu plus lentement que la locutrice native: son débit est de 3,8 syllabes par seconde dans cet extrait, tandis que la locutrice native parle à une vitesse de 4,5 syllabes par seconde. ${ }^{15}$ La moyenne de la $f_{0}$ est de $195,5 \mathrm{~Hz}$ dans la parole de la locutrice finnophone et de $250,6 \mathrm{~Hz}$ dans la parole de la

\footnotetext{
${ }^{14}$ Pour chaque énoncé interrogatif, nous présentons un exemple de la parole d'un apprenant finnophone et un exemple de la parole d'un locuteur natif. Les personnes ne sont pas toujours les mêmes.

${ }^{15}$ Selon Morange (2005), le débit moyen en français est de 5,0 syllabes par seconde.
} 


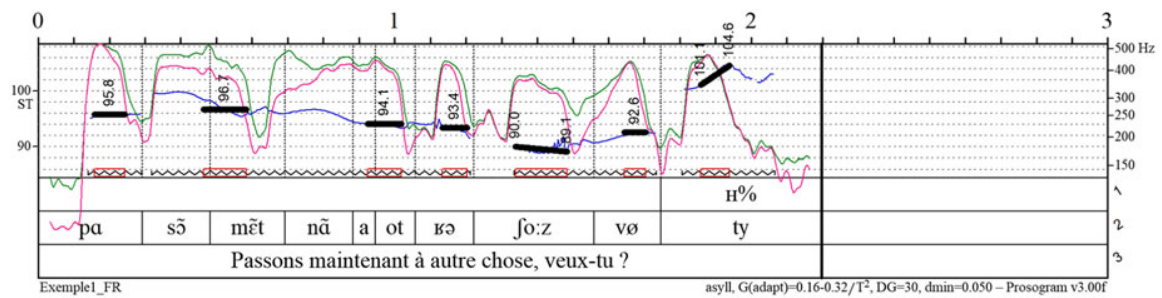

Figure 2. Question totale "Passons maintenant à autre chose, veux-tu ? produite par une locutrice native du français.

locutrice native du français. Les deux valeurs sont typiques d'une voix féminine. L'écart-type de la $f_{0}$ est de 3,6 dt dans la parole de la locutrice finnophone et de $6,3 \mathrm{dt}$ dans la parole de la locutrice native du français. De ce fait, il y a une différence intéressante à noter entre les deux informatrices: bien que l'étendue $\mathrm{du}$ mouvement intonatif final soit similaire dans les deux cas, il y a plus de variations mélodiques dans la parole de la locutrice native que dans celle de l'apprenante finnophone du français.

Il est bien connu que l'inversion du sujet clitique est utilisée surtout à l'écrit, en particulier dans les textes littéraires (Riegel, Pellat et Rioul, 1994:392), mais qu'elle s'utilise aussi à l'oral avec certains verbes irréguliers les plus fréquents (Coveney, 2011: 15). C'est le cas par exemple dans la réplique d'Estragon «Passons maintenant à autre chose, veux-tu ?» (Beckett, 1952: 118). L'inversion du sujet s'utilise aussi pour permettre au locuteur d'utiliser un registre plus formel. D'autre part, "veux-tu ?» est aussi un élément du discours, et pas seulement une interrogation. Coveney $(2013: 2)$ signale à ce sujet que:

Dans des études quantitatives des différentes variantes, il est clair que les formes lexicalisées ne devraient pas être comptées comme des interrogatives : par exemple, les substantifs qu'en dira-t-on? ou m'as-tu vu?, et les marqueurs de discours n'estce pas?, voyez-vous?, tu vois? (Coveney, 2013: 2).

Effectivement, les questions de Beckett qui se terminent en formes lexicalisées interrogatives, ne sont pas à proprement parler des questions totales puisqu'on y répondrait difficilement par 'oui' ou par 'non'. Elles réclament plutôt une réponse plus longue qui répète en partie ce que le locuteur vient de dire ou une réponse qui est déjà anticipée par le locuteur.

Le contexte d'occurrence de la question «Passons maintenant à autre chose, veux-tu? ? est présenté ci-dessous (Exemple 1).

\section{Exemple 1:}

01 Vladimir. - Eh bien ? (Un temps.) Qu'est-ce qu'il a de si

02 extraordinaire?

03 Silence.

04 Estragon. - Passons maintenant à autre chose, veux-tu?

05 Vladimir. - J'allais justement te le proposer. 
La question d'Estragon (ligne 04) est précédée d'un silence (ligne 03), ce qui peut éventuellement jouer un rôle sur le plan prosodique: autrement dit, le fait que la question rompt le silence peut créer une nécessité de la mettre en valeur en produisant une montée intonative. En effet, sur les deux pages, ce sont les mêmes didascalies, à savoir "Silence » et "Un temps » qui reviennent. La première revient quatre fois, la seconde deux fois, ce qui veut dire que la communication entre les trois personnages est interrompue, de façon répétitive, par celui qui n'écoute pas ou par celui qui prend son temps à réfléchir avant de répondre. Lorsque Vladimir, Estragon ou Pozzo lancent une question à leur interlocuteur, la question s'adresse à eux-mêmes aussi. C'est un fait de style qui est propre à l'écriture beckettienne et qui peut effectivement avoir une certaine influence sur la lecture des locuteurs natifs qui sont familiers avec le style minimaliste de l'auteur.

\subsubsection{Exemple 2: "Ça va mieux?»}

Ce type de question, construite sans marqueur lexical ou morpho-syntaxique d'interrogation, s'utilise beaucoup dans le français parlé. D’après Coveney (2011: 12), la structure 'sujet' + 'verbe' + 'intonation montante' est de loin la structure la plus fréquente pour les questions totales. La figure 3 présente la forme acoustique de l'énoncé interrogatif «Ça va mieux? » produit par une apprenante finnophone du français.

Comme la figure 3 le montre bien, l'intonation monte très clairement à la fin de la question: l'étendue de la montée est de 11,7 dt. Il s'agit donc d'un contour extramontant (HH\%). La moyenne de la $f_{0}$ est de $213,4 \mathrm{~Hz}$, ce qui correspond à une valeur moyenne pour une femme. L'écart-type de la $f_{0}$ est de $4,0 \mathrm{dt}$. Le débit est de 4,3 syllabes par seconde.

La figure 4 illustre la forme acoustique de la même question produite par une locutrice native du français.

La locutrice native du français produit aussi un contour extra-montant ( $\mathrm{HH} \%)$ à la fin de cette question totale. L'intonation monte $13,4 \mathrm{dt}$ pendant la production de la dernière syllabe accentuée de l'énoncé ([mjø]). La moyenne de la $f_{0}$ est de 245,4 $\mathrm{Hz}$. L'écart-type de la $f_{0}$ est de $4,0 \mathrm{dt}$, ce qui est exactement autant que dans la parole de l'apprenante finnophone. Le débit est de 5,0 syllabes par seconde. De ce fait, il n'y a pas de différences remarquables à noter en ce qui concerne les traits prosodiques de cette question dans la parole de la locutrice finnophone et dans celle de la locutrice native.

Le contexte d'occurrence de la question «Ça va mieux? » est donné ci-dessous (Exemple 2):

\section{Exemple 2:}

01 Vladimir. - Il veut qu'on l'aide à se lever.

02 Estragon. - Eh bien, aidons-le. Qu'est-ce qu'on attend?

$03 \mathrm{Ils}$ aident Pozzo à se lever, s'écartent de lui. Il retombe.

04 Vladimir. - Il faut le soutenir (Même jeu. Pozzo reste debout entre les

06 deux, pendu à leur cou.) Il faut qu'il se réhabitue à la station

07 Pozzo. - Qui êtes-vous? 


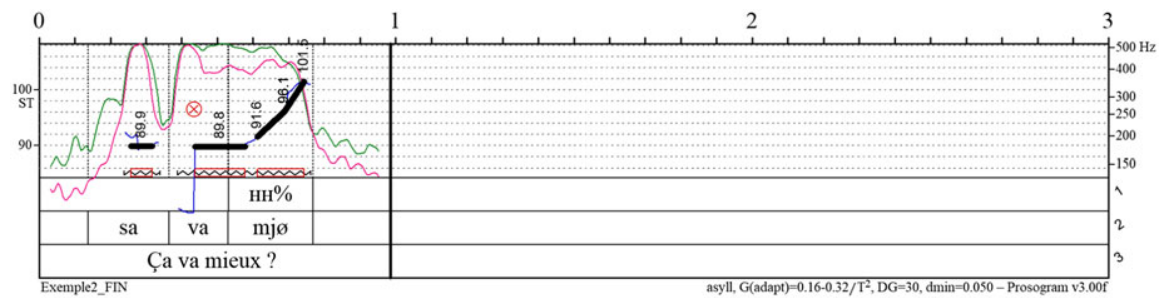

Figure 3. Question totale «Ça va mieux?» produite par une apprenante finnophone du français.

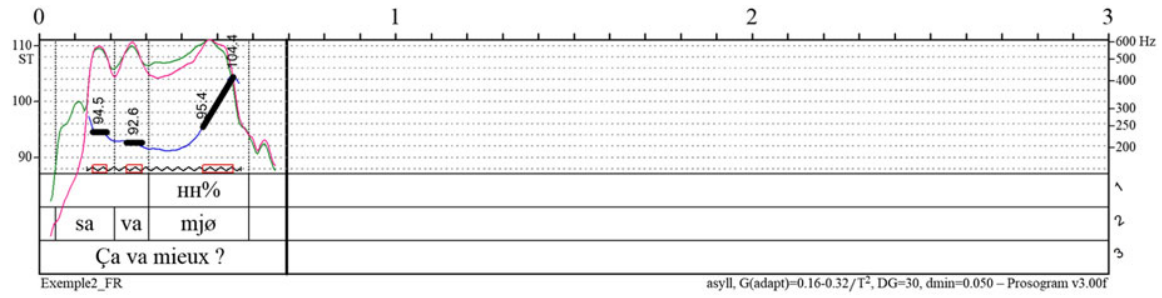

Figure 4. Question totale « Ça va mieux? » produite par une locutrice native du français.

La question apparaît ici à la fin du tour de parole de Vladimir (ligne 06). Estragon et Vladimir sont en train d'aider Pozzo à se lever, mais il a du mal à rester debout. En plus d'indiquer l'interrogation, la forte montée intonative apparaissant relativement souvent à la fin de cet énoncé peut avoir une fonction expressive : autrement dit, elle peut par exemple servir à transmettre une nuance d'inquiétude.

\subsection{Questions partielles}

En ce qui concerne les questions partielles, les résultats sont plus divergents que les résultats concernant les questions totales. Le plus souvent (56\%), les apprenants finnophones produisent un contour montant $(\mathrm{H} \%)$ à la fin d'une question partielle. Il y a aussi des occurrences du contour extra-montant (HH\%, 13\%) dans la parole des apprenants L2. La proportion des montées finales (les types $\mathrm{H}$ $\%$ et $\mathrm{HH} \%$ inclus) y est de $69 \%$. Dans la parole des locuteurs natifs du français, en revanche, l'intonation descendante (L\%, 40\%) et plate $(0 \%, 33 \%)$ sont nettement plus fréquentes que l'intonation montante: la proportion des contours descendant (L\%) et plat $(0 \%)$ est de $73 \%$. De ce fait, il y a une différence très claire à noter entre les deux groupes d'informateurs en ce qui concerne l'intonation des questions partielles: les apprenants finnophones ont tendance à produire une intonation montante à la fin des questions partielles, tandis que les locuteurs natifs produisent plutôt une intonation descendante ou plate dans cette position.

Les résultats concernant le groupe de contrôle sont conformes aux études antérieures selon lesquelles les questions partielles neutres se terminent typiquement par une chute intonative finale (L\%) en français (Delais-Roussarie 
Tableau 2. Questions partielles: types du ton de frontière et valeurs de l'étendue moyennes des mouvements mélodiques finals. Apprenants finnophones (FIN) et locuteurs natifs (FR)

\begin{tabular}{|c|c|c|c|c|}
\hline Question & $\begin{array}{l}\text { Type du ton } \\
\text { de frontière } \\
\text { (FIN), nombre } \\
\text { d'occurrences }\end{array}$ & $\begin{array}{l}\text { Étendue moyenne } \\
\text { du mouvement } \\
\text { mélodique final } \\
\text { (dt) (FIN) }\end{array}$ & $\begin{array}{l}\text { Type du ton } \\
\text { de frontière } \\
\text { (FR), nombre } \\
\text { d'occurrences }\end{array}$ & $\begin{array}{l}\text { Étendue moyenne du } \\
\text { mouvement } \\
\text { mélodique final (dt) } \\
\text { (FR) }\end{array}$ \\
\hline $\begin{array}{l}\text { «Qu'est-ce qu'il y a } \\
\text { de si extraordi- } \\
\text { naire?» }\end{array}$ & $\begin{array}{l}\mathrm{H} \%: 6 \\
\mathrm{~L} \%: 4 \\
\mathrm{O} \%: 3 \\
\mathrm{HH} \%: 2\end{array}$ & $\begin{array}{l}\mathrm{H} \%:+6,7 \mathrm{dt} \\
\mathrm{L} \%:-8,8 \mathrm{dt} \\
0 \%:-1,1 \mathrm{dt} \\
\mathrm{HH} \%:+11,0 \mathrm{dt}\end{array}$ & $\begin{array}{l}\mathrm{H} \%: 1 \\
\mathrm{~L} \%: 1 \\
0 \%: 3\end{array}$ & $\begin{array}{l}\mathrm{H} \%:+3,2 \mathrm{dt} \\
\mathrm{L} \%:-4,5 \mathrm{dt} \\
0 \%:+1,3 \mathrm{dt}\end{array}$ \\
\hline «Pourquoi?» & $\begin{array}{l}\mathrm{H} \%: 13 \\
\mathrm{~L} \%: 1 \\
\mathrm{HH} \%: 1\end{array}$ & $\begin{array}{l}\mathrm{H} \%:+5,1 \mathrm{dt} \\
\mathrm{L} \%:-7.7 \mathrm{dt} \\
\mathrm{HH} \%:+11.6 \mathrm{dt}\end{array}$ & $\begin{array}{l}\mathrm{H} \%: 2 \\
\mathrm{~L} \%: 1 \\
0 \%: 2\end{array}$ & $\begin{array}{l}\mathrm{H} \%:+3,6 \mathrm{dt} \\
\mathrm{L} \%:-10.1 \mathrm{dt} \\
0 \%:+1,0 \mathrm{dt}\end{array}$ \\
\hline «Qui êtes-vous?» & $\begin{array}{l}\mathrm{H} \%: 6 \\
\mathrm{~L} \%: 4 \\
0 \%: 2 \\
\mathrm{HH} \%: 3\end{array}$ & $\begin{array}{l}\mathrm{H} \%: 7,3 \mathrm{dt} \\
\mathrm{L} \%:-6,5 \mathrm{dt} \\
0 \%:-1,4 \mathrm{dt} \\
\mathrm{HH} \%:+14,6 \mathrm{dt}\end{array}$ & $\begin{array}{l}\mathrm{H} \%: 1 \\
\mathrm{~L} \%: 4\end{array}$ & $\begin{array}{l}\mathrm{H} \%:+6.8 \mathrm{dt} \\
\mathrm{L} \%:-5,0 \mathrm{dt}\end{array}$ \\
\hline
\end{tabular}

et al., 2015; Di Cristo, 1998 : 205). Les résultats concernant le groupe d'apprenants finnophones du français, quant à eux, sont similaires à ceux de Santiago et DelaisRoussarie (2015: 267) selon lesquels les apprenants hispanophones du français emploient un contour montant dans la plupart des questions partielles $(80 \%)$. De plus, le contour extra-montant (HH\%) y est plus souvent utilisé par les apprenants L2 que par les locuteurs natifs aussi bien dans nos données que dans celles de Santiago et Delais-Roussarie (2015). Les nombres d'occurrences de différents tons de frontière ainsi que les valeurs de l'étendue moyennes des mouvements mélodiques concernant les questions partielles sont donnés dans le tableau 2.

\subsubsection{Exemple 3: "Qu'est-ce qu'il y a de si extraordinaire?»}

Sur les cinq phrases qui ont été retenues pour l'analyse, une est construite avec la locution 'est-ce que' : "Qu'est-ce qu'il y a de si extraordinaire?». Précédé du pronom interrogatif 'que', c'est un énoncé interrogatif partiel qui ne s'interroge que sur une partie de la phrase (Riegel, Pellat et Rioul, 1994: 391-401). Contrairement aux interrogations totales auxquelles il suffit de répondre par «oui» ou «non», les questions partielles réclament comme réponse une unité lexicale (Gardes-Tamine, 2004 : 37).

Comme nous l'avons déjà constaté plus haut, les questions construites avec 'estce que' se terminent systématiquement en un contour clairement descendant (L\%) dans le corpus de Delais-Roussarie et al. (2015). Cela n'est pas étonnant, puisque la construction 'est-ce que', en tête de phrase, indique déjà qu'il s'agit d'une question. Par conséquent, l'intonation montante perd sa nécessité. Pour les apprenants finnois de FLE, ce type de question est bien connu. Les manuels scolaires finlandais le pratiquent beaucoup (Tyni, 2021), puisque l'interrogation avec 'estce que' a le double avantage de permettre le maintien de l'ordre canonique ainsi 


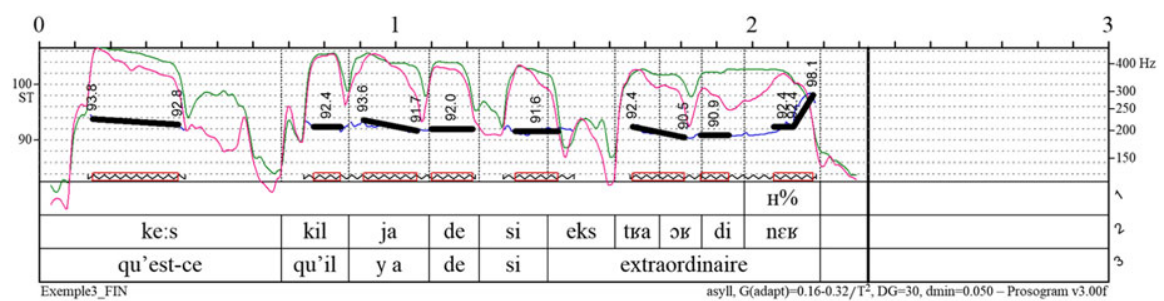

Figure 5. Question partielle «Qu'est-ce qu'il y a de si extraordinaire? » produite par une apprenante finnophone du français.

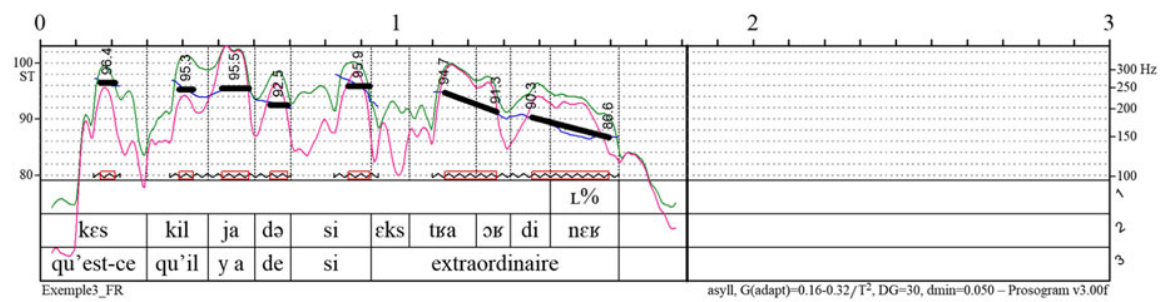

Figure 6. Question partielle «Qu'est-ce qu'il y a de si extraordinaire ? » produite par une locutrice native du français.

que de fournir d'emblée une marque de l'interrogation, ce qui permet d'éviter l'intonation montante à la fin de l'énoncé. ${ }^{16}$ Les deux - l'ordre canonique et l'intonation descendante / plate - sont considérés comme étant plus faciles pour les apprenants finnophones de FLE.

La figure 5 présente la forme acoustique de l'énoncé interrogatif «Qu'est-ce qu'il y a de si extraordinaire?» produite par une apprenante finnophone du français.

Comme on peut le voir dans la figure 5, l'intonation monte clairement à la fin de l'énoncé interrogatif, au moment de la production de la dernière syllabe accentuée ([nєь]). L'étendue de la montée est de 7,7 dt. Il s'agit donc d'un contour montant du type $\mathrm{H} \%$. À part la montée finale, l'intonation est monotone dans cet échantillon de parole: en effet, l'écart-type de la $f_{0}$ y est seulement de $1,4 \mathrm{dt}$. La moyenne de la $f_{0}$ est de $206,3 \mathrm{~Hz}$, ce qui est un niveau de la $f_{0}$ moyenne pour une femme. Le débit est de 4,3 syllabes par seconde.

La forme acoustique de la même question produite par une locutrice native du français est donnée dans la figure 6.

Le contour intonatif est descendant (L\%) dans l'échantillon de parole présentée dans la figure 6 : l'intonation descend 4,8 dt entre la syllabe pénultième ([di]) et la dernière syllabe accentuée ([nєr]). La moyenne de la $f_{0}$ est de 213,4 Hz. L'écart-type est de 3,1 dt. Cette valeur est supérieure à l'écart-type de la locutrice finnophone, mais elle correspond malgré tout à une échelle de variations relativement restreinte. Le débit est de 5,6 syllabes par seconde. La locutrice native parle donc plus vite que l'apprenante finnophone, mais son débit n'est pas particulièrement rapide non plus.

\footnotetext{
${ }^{16}$ Voir à ce sujet par exemple Riegel, Pellat et Rioul (1994: 393).
} 
L'extrait ci-dessous (Exemple 3) illustre le contexte d'occurrence de la question "Qu'est-ce qu'il a de si extraordinaire?».

\section{Exemple 3:}

01 Estragon. - C'est toute l'humanité. (Silence.) Regarde-moi ce petit

02 nuage.

03 Vladimir (levant les yeux). - Où?

04 Estragon. - Là, au zénith.

05 Vladimir. - Eh bien? (Un temps.) Qu'est-ce qu'il a de si

06 extraordinaire?

07 Silence.

08 Estragon. - Passons maintenant à autre chose, veux-tu?

La question «Qu'est-ce qu'il a de si extraordinaire?» est probablement une question rhétorique. Dans cette scène, Vladimir (se) demande ce qu'il y a de si extraordinaire dans le petit nuage mentionné par Estragon, mais il n'attend pas nécessairement de réponse à sa question. En effet, Estragon n'y répond pas, mais la question est suivie d'un silence (ligne 07). Que l'informateur ait interprété cette interrogation en tant que question rhétorique peut effectivement exercer une certaine influence sur la forme prosodique de l'énoncé. La plupart des locuteurs natifs de notre corpus (4/5) l'ont produite sans montée finale, et même dans la parole des apprenants finnophones, il y a beaucoup de variation individuelle en ce qui concerne la forme du contour final. Cette variation individuelle peut cependant avoir aussi d'autres explications qui n'ont pas de rapport avec la question rhétorique. Parmi ces explications se trouve sans doute la difficulté que plusieurs locuteurs finnophones ont rencontrée à prononcer l'adjectif qualificatif 'extraordinaire'.

\subsubsection{Exemple 4: «Pourquoi?»}

L'extrait de Beckett (1952: 118-119) est riche en formes elliptiques qui servent, elles aussi, à poser des questions. Beckett emploie ces formes avec ou sans mot interrogatif. Leur emploi est abondant puisqu'on trouve huit occurrences dans notre extrait de deux pages. A l'époque de Beckett, les grammairiens parlaient dans ce cas-là de la 'phrase simple' (Grammaire du français contemporain 1964 : 62) alors qu'actuellement, on les appelle plutôt des 'phrases atypiques' ou 'nominales' (Lefeuvre, 1999). Réduites à un mot (interrogatif ou non), ces questions sont parfois précédées d'une conjonction ou d'un adverbe.

En ce qui concerne la réplique «Pourquoi ? » d'Estragon (Beckett 1952:118), 14/ 15 informateurs finnophones (93\%) la produisent soit avec un contour montant $(\mathrm{H} \%$, $13 / 15)$ soit avec un contour extra-montant $(\mathrm{HH} \%, 1 / 15)$. Un informateur finnophone produit un contour descendant (L\%). Les résultats concernant le groupe des locuteurs natifs du français sont plus variés : $2 / 5$ informateurs produisent un contour montant $(\mathrm{H}$ $\%), 2 / 5$ produisent un contour plat $(0 \%)$, et $1 / 5$ produit un contour descendant (L\%). De ce fait, il est plus fréquent $(3 / 5,60 \%)$ pour les locuteurs natifs de produire un contour plat ou descendant qu'un contour montant dans le cas de cette question. L'étendue moyenne des montées produites par les locuteurs natifs $(3,6 \mathrm{dt})$ est aussi plus modeste que celle des montées produites pas les apprenants finnophones $(5,1 \mathrm{dt})$. 


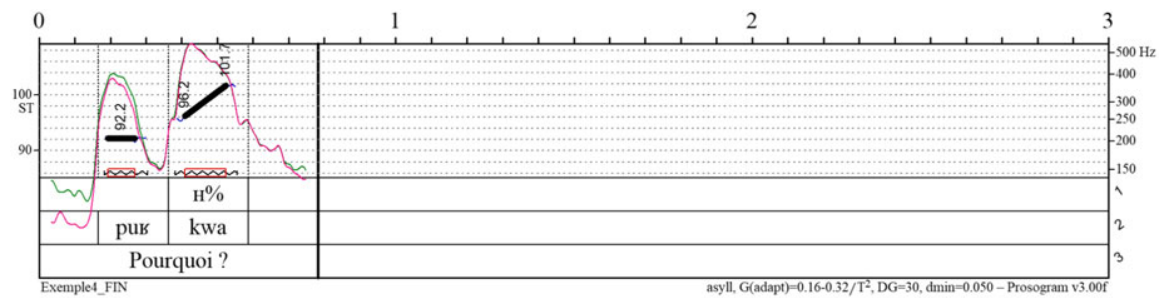

Figure 7. Question partielle «Pourquoi?»produite par une apprenante finnophone du français.

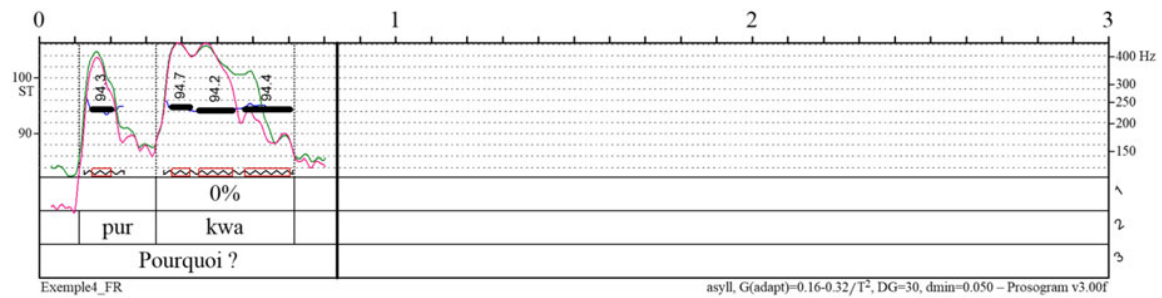

Figure 8. Question partielle «Pourquoi ? » produite par une locutrice native du français.

La figure 7 illustre la forme acoustique de la question «Pourquoi ? » produite par une apprenante finnophone du français.

Comme la figure 7 le montre bien, l'intonation monte clairement à la fin de la question: l'étendue de la montée est de 6,4 dt. Il s'agit donc d'un contour montant du type $\mathrm{H} \%$. La moyenne de la $f_{0}$ est de $266,6 \mathrm{~Hz}$, et l'écart-type de la $f_{0}$ est de 3,8 dt. Le débit est de 5,0 par seconde.

La figure 8 présente la forme acoustique de la question «Pourquoi? » produite par une locutrice native du français.

Comme on peut le voir dans la figure 8 , l'intonation est vraiment plate $(0 \%)$ lors de la production de cette question par une locutrice native du français : l'intonation baisse de $0,1 \mathrm{dt}$ lors de la production de la dernière syllabe du mot par rapport à la première syllabe. La valeur moyenne de la $f_{0}$ est de $234,2 \mathrm{~Hz}$. L'écart-type est seulement de $0,5 \mathrm{dt}$, ce qui est remarquablement peu. Le débit est seulement de 3,3 syllabes par seconde, ce qui correspond à un débit lent.

Le contexte d'occurrence de la question «Pourquoi ?» est présenté dans l'extrait ci-dessous (Exemple 4):

\section{Exemple 4:}

01 Estragon. - Et maintenant?

02 Pozzo. - Au secours!

03 Estragon. - Allons-nous-en.

04 Vladimir. - On ne peut pas.

05 Estragon. - Pourquoi ?

06 Vladimir. - On attend Godot.

07 Estragon. - C'est vrai. (Un temps.) Que faire? 
La question «Pourquoi? » (ligne 05), posée par Estragon, apparaît ici dans une suite de répliques courtes, ce qui peut avoir une influence sur sa forme prosodique. Il s'agit aussi d'une réaction à la réplique de Vladimir, "On ne peut pas.» (ligne 04). Par conséquent, la forme prosodique de la question peut être liée aussi à l'expression d'étonnement. Chez Beckett, les interrogations ont la particularité de se fusionner souvent avec des exclamations. Autrement dit, l'expression de l'interrogation ne se sépare pas vraiment de l'expression d'une attitude ni d'une émotion dans la communication entre Vladimir, Estragon et Pozzo.

\subsubsection{Exemple 5: "Qui êtes-vous?»}

Les questions du type «Qui êtes-vous? » ne sont pas rares à l'oral; bien au contraire, l'inversion résiste bien à l'oral. L'interrogation dans cette réplique de Pozzo (Beckett 1952 : 119) est partielle, car elle ne porte que sur une chose, à savoir l'identité de la personne. Comme le pronom interrogatif et l'inversion du sujet clitique permettent au locuteur de comprendre d'emblée qu'il s'agit d'une question, l'intonation montante y perd sa nécessité. En effet, dans nos données, 4/5 locuteurs natifs du français $(80 \%)$ produisent cette question avec un contour descendant (L\%); seulement un locuteur natif la produit avec une intonation montante $(\mathrm{H} \%)$. Quant aux apprenants finnophones du français, les résultats sont plus variés : 6/ 15 produisent un contour montant $(\mathrm{H} \%), 4 / 15$ produisent un contour descendant (L\%), 3/15 produisent un contour extra-montant (HH\%) et 2/15 produisent un contour plat (0\%). La proportion des informateurs finnophones qui produisent une intonation montante $(\mathrm{H} \%$ ou $\mathrm{HH} \%)$ est de $60 \%$, ce qui est nettement plus que la proportion correspondante parmi les informateurs natifs $(20 \%)$.

La figure 9 présente la forme acoustique de la question «Qui êtes-vous?» produite par une apprenante finnophone du français.

L'intonation monte très clairement lors de la production de la dernière syllabe ([vu]) de la question (Figure 9) : l'étendue de la montée est de 10,9 dt. Il s'agit donc d'un contour extra-montant (HH\%). La moyenne de la $f_{0}$ est de $282,8 \mathrm{~Hz}$. L'écart-type de la $f_{0}$ est de 4,4 dt. Le débit est de 5,0 syllabes par seconde.

La figure 10 présente la forme acoustique de la même question produite par une locutrice native du français.

Comme la figure 10 le montre bien, la locutrice produit un contour descendant (L $\%$ ) à la fin de cette question : l'intonation baisse de 9,9 dt entre la syllabe pénultième $([\varepsilon t])$ et la dernière syllabe $([v u])$. La perception de l'intonation est clairement descendante. La moyenne de la $f_{0}$ est de $248,1 \mathrm{~Hz}$. L'écart-type de la $f_{0}$ est de 3,5 $\mathrm{dt}$, ce qui est un peu moins que dans l'échantillon de parole de l'apprenante finnophone. Le débit est de 4,3 syllabes par seconde - aussi un peu moins que dans la parole de l'apprenante L2. ${ }^{17}$

\footnotetext{
${ }^{17}$ À titre illustratif, nous avons indiqué aussi le débit des échantillons de parole présentés dans l'article, mais le débit n’a pas été analysé systématiquement dans cette étude. Par conséquent, nous ne préférons pas faire de conclusions du rôle de débit dans ces données. Néanmoins, il serait très intéressant d'étudier le débit plus en détail à l'avenir, puisque les changements de débit dans ces données sont probablement liés à l'interprétation du texte littéraire.
} 


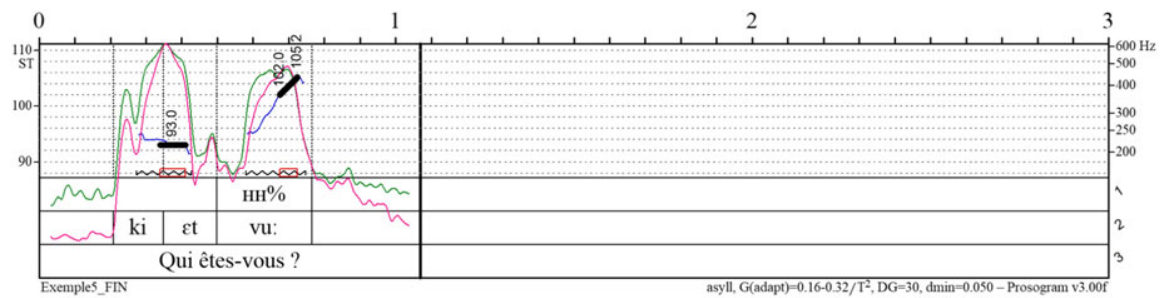

Figure 9. Question partielle «Qui êtes-vous ? produite par une apprenante finnophone du français.

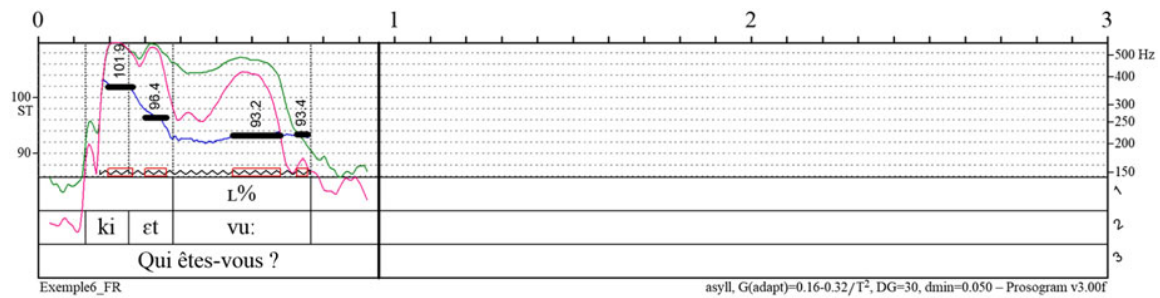

Figure 10. Question partielle «Qui êtes-vous ?» produite par une locutrice native du français.

L'extrait ci-dessous (Exemple 5) illustre le contexte d'occurrence de la question «Qui êtes-vous?».

\section{Exemple 5:}

$01 \mathrm{Ils}$ aident Pozzo à se lever, s'écartent de lui. Il retombe.

02 Vladimir. - Il faut le soutenir (Même jeu. Pozzo reste debout entre les

03 deux, pendu à leur cou.) Il faut qu'il se réhabitue à la station debout. (A 04 Pozzo.)

Ça va mieux?

05 Pozzo. - Qui êtes-vous?

06 Vladimir. - Vous ne nous remettez pas?

07 Pozzo. - Je suis aveugle.

La question de Pozzo, "Qui êtes-vous? ( (ligne 05) est une réaction à la question «Ça va mieux? " posée par Vladimir (ligne 04). Pozzo ne répond donc pas à la question de Vladimir, mais il y réagit en posant lui-même une question très directe. La question de Pozzo n'est pas nécessairement complètement neutre sur le plan émotionnel, mais il peut véhiculer par exemple une nuance de peur. Cette nuance émotionnelle peut avoir un effet sur la forme prosodique de la question - autrement dit, sur le fait que la plupart des informateurs natifs la produisent avec une intonation descendante, et même dans la parole des apprenants finnophones, il y a beaucoup de variation individuelle. Comment interpréter cette variation ? Il nous semble qu'elle est liée à l'incertitude des locuteurs finnophones qui ne sont pas encore à l'aise, ni familiers avec l'intonation expressive du français. 


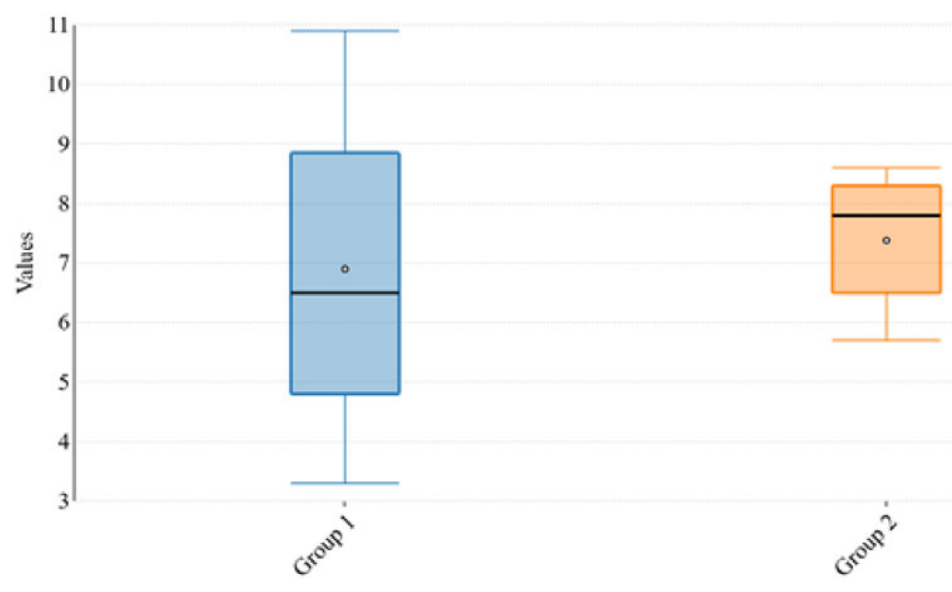

Figure 11. Diagramme en boîte à moustaches illustrant la répartition des données. 'Group 1' : apprenants finnophones du français. 'Group 2' : locuteurs natifs du français.

\subsection{Valeurs de l'étendue des montées intonatives}

Pour calculer les moyennes et les écarts-types des valeurs de l'étendue des contours montants (les types $\mathrm{H} \%$ et $\mathrm{HH} \%$ inclus) apparaissant à la fin des questions totales et des questions partielles dans les deux groupes d'informateurs, un test de ShapiroWilk a été mené. Le même test a été utilisé pour vérifier que les données analysées étaient normalement distribuées. Les résultats montrent que l'étendue moyenne des montées est de 6,9 dt pour les apprenants finnophones et de 7,4 dt pour les locuteurs natifs. L'écart-type est de $2,7 \mathrm{dt}$ pour les apprenants finnophones et de 1,2 pour les locuteurs natifs. Autrement dit, il y a plus de variation individuelle dans les valeurs de l'étendue des apprenants finnophones que dans celles des locuteurs natifs. Les données sont normalement distribuées.

Pour comparer les moyennes des deux groupes d'informateurs, un test- $t$ de Welch a été mené. Les résultats de ce test statistique - qui est une adaptation du test-t de Student pouvant être utilisé notamment pour comparer les moyennes des échantillons de variances inégales ou de taille différentes - montre que la différence entre les moyennes des deux groupes d'informateurs n'est pas statistiquement significative (la valeur- $p=0,577736$ ). Le diagramme en boîte à moustaches (Figure 11) illustre la répartition des données dans les deux groupes d'informateurs.

\section{EXPLICATION DES DIFFÉRENCES ET QUELQUES IMPLICATIONS PÉDAGOGIQUES}

La fréquence des contours montants dans la parole des apprenants finnophones est probablement liée au fait que l'intonation montante à la fin des énoncés interrogatifs est mise en évidence dans l'enseignement du FLE. Pour justifier notre interprétation, nous avons comparé la présentation des phrases interrogatives dans trois manuels de FLE (Escalier 1, J'aime 1 et Cosmopolite 1) utilisés en Finlande en 2018. 
L'explication minutieuse des caractéristiques de l'écrit domine au détriment des énoncés interrogatifs qui relèveraient du langage parlé dans Escalier 1. Le livre prend soin et choisit d'expliquer l'interrogation avec inversion et va jusqu'à expliquer l'emploi du $t$ intercalé ("Quelles langues parle-t-on en Suisse?») sans commenter le rôle de l'intonation en dehors des questions construites avec intonation (p. 91 et p. 250). Contrairement à Escalier 1, J'aime 1 s'intéresse plutôt aux caractéristiques des questions qui relèvent du langage parlé. Mais malheureusement, celui-ci laisse entendre que l'affaire est manichéenne et se contente de sensibiliser les apprenants à identifier l'intonation montante («Ça va?») en tant que caractéristique essentielle permettant de distinguer rapidement les interrogations des affirmations («Ça va.») (p. 21).

L'avantage du manuel français Cosmopolite 1 est d'introduire l'intonation montante en tant qu'élément qui est propre aux phrases exclamatives permettant l'expression des affects et des attitudes (p. 139). Il nous semble que c'est la bonne voie. Enseigner la phrase interrogative et la phrase exclamative ensemble ne serait pas seulement logique mais aussi éthique, car il est important d'enseigner à l'heure actuelle la fonction affective de l'intonation dans l'expression des attitudes et des émotions. Et ce, parce que malgré le fait que la pratique de l'oral est d'ores et déjà mise en valeur dans l'enseignement des langues étrangères, on aurait besoin de méthodes avec lesquelles on pourrait, de façon plus efficace, enseigner l'emploi de l'intonation de façon à permettre aux apprenants de mieux s'exprimer et de se faire comprendre en une langue étrangère. Nous sommes en effet d'accord avec les spécialistes de FLE qui trouvent que:

Communiquer est le moyen; s'entendre est le but. Le monde actuel a probablement plus besoin d'humanité que de langues, ou plus précisément a-t-il besoin d'humanité au travers de ses diverses langues et des échanges authentiques qu'elles permettent (Defays, Delbart, Hammami et Saenen, 2014: 19).

Quelles sont les autres implications de notre étude pour l'enseignement du français comme langue étrangère? Nous avons revu que - comme il est déjà bien connu si un marqueur lexical d'interrogation est utilisé, la montée finale n'a pas besoin d'être très haute, puisque l'interlocuteur (francophone natif) comprend déjà par ce marqueur lexical qu'une question lui a été posée. Nous avons également vu que si l'intonation montante perd sa nécessité, elle a tendance à être remplacée par une intonation plate. Pédagogiquement, c'est une information utile et utilisable que nous avons découverte même si ce n'est pas une information nouvelle. En effet, la structure syntaxique et lexicale de la question affecte toujours plus ou moins son intonation mais, d'après notre étude, cette information devrait être mise en évidence de façon explicite aussi dans les manuels de FLE, ce qui n'est pas le cas à l'heure actuelle. Elle devrait être impliquée dans l'enseignement de façon à faire comprendre aux apprenants de FLE que le français supporte mal la redondance. Cela ne vaut pas seulement pour la rédaction d'un texte ou pour la maitrise des règles de grammaire mais aussi pour l'intonation des énoncés interrogatifs. De la même manière que l'adverbe 'beaucoup' ne doit pas être renforcé par l'adverbe 'très', il suffit d'avoir un élément interrogatif dans l'énoncé interrogatif. Le mot interrogatif 
'pourquoi', par exemple, est un marqueur d'interrogation extrêmement fort exprimant déjà par sa sémantique l'interrogation; l'intonation montante serait-elle alors l'expression d'autre chose - l'expression d'un sentiment d'étonnement du locuteur par exemple?

\section{CONCLUSION}

Nos résultats montrent qu'aussi bien les apprenants finnophones du français que les locuteurs natifs du français produisent une montée intonative à la fin des questions totales d'information. La montée peut être soit un contour montant $(\mathrm{H} \%, \leq 10 \mathrm{dt})$ soit un contour extra-montant $(\mathrm{HH} \%,>10 \mathrm{dt})$. Ce résultat est conforme aux études antérieures selon lesquelles les questions totales d'information du français se terminent typiquement en une montée intonative portée par la dernière syllabe accentuée de l'énoncé (Delais-Roussarie et al., 2015; Di Cristo, 1998: 204). En ce qui concerne les questions partielles, les résultats sont plus divergents. Le plus souvent, les apprenants finnophones produisent aussi une montée intonative à la fin d'une question partielle: la proportion des montées finales (les types $\mathrm{H} \%$ et $\mathrm{HH} \%$ inclus) est de 69\%. Dans la parole des locuteurs natifs du français, en revanche, l'intonation descendante et plate sont nettement plus fréquentes que l'intonation montante: la proportion des contours descendants (L\%) et plats (0\%) est de $73 \%$. De ce fait, il y a une différence très claire à noter entre les deux groupes d'informateurs en ce qui concerne l'intonation des questions partielles: les apprenants finnophones ont tendance à produire aussi une intonation montante à la fin des questions partielles, tandis que les locuteurs natifs produisent plutôt une intonation descendante ou plate dans cette position.

Pour ce qui est de la comparaison des valeurs de l'étendue des montées intonatives dans les deux groupes d'informateurs, il n'y a pas de différences à noter. En effet, lorsque l'on compte tous les contours montants et contours extra-montants de notre corpus, l'étendue moyenne des montées est de 6,9 dt pour les apprenants finnophones et de 7,4 dt pour les locuteurs natifs. La différence n'est pas statistiquement significative.

Étant donné qu'en finnois, le contour descendant concerne d'une manière générale aussi bien les assertions que les questions (Iivonen, 1998: 317), le fait que les apprenants finnophones du français terminent typiquement toutes les questions en une montée intonative $(\mathrm{H} \%$ ou $\mathrm{HH} \%)$ ne peut pas s'expliquer par l'activation de la transmission des traits prosodiques de la L1 à la L2. Probablement, il ne s'agit pas non plus ici de l'association universelle des contours montants à la fonction interrogative (Gussenhoven, 2004), puisque cette "universalité » ne concerne donc pas le finnois (Iivonen, 1998), qui est la langue maternelle des apprenants L2 de notre étude. L'incertitude linguistique des apprenants L2 (Horgue, 2010; Santiago et Delais-Roussarie, 2015) peut éventuellement jouer un rôle dans ce cas, puisque nos informateurs sont des débutants du niveau A1-A2 (CEFR, 2021). Il est également possible que la fréquence des contours montants dans la parole des apprenants finnophones soit expliquée en partie par l'interférence avec l'anglais qui est une langue que l'on entend beaucoup dans les médias finlandais.

Nos analyses suggèrent cependant que la fréquence des contours montants et des contours extra-montants est liée surtout au fait que dans l'enseignement du FLE en 
Finlande, on souligne l'importance de monter l'intonation à la fin des énoncés interrogatifs (Tyni, 2021) sans expliquer les différences concernant l'étendue de l'intonation et les effets souvent exclamatifs de celles-ci. En effet, sur la base des manuels de FLE dont nous avons comparé la présentation des phrases interrogatives, il est possible d'arguer sur la fonction du contour montant à la fin des énoncés interrogatifs la suivante : les manuels finlandais (J'aime 1 et Escalier 1) laissent entendre que ce sont seules les interrogations construites avec intonation qui nécessitent une montée intonative. Les explications plus nuancées qui illustraient la signification et l'expressivité des chutes et des montées intonatives, dont l'étendue peut varier selon la situation et l'attitude du locuteur, y manquent. Par conséquent, face à cette lacune, l'apprenant tire ses propres conclusions, ce qui expliquerait, du moins en partie, la variation individuelle dans la parole des apprenants finnophones.

En outre, et en contraste avec les manuels de FLE, nous avons vu que chez Beckett, les interrogations ont la particularité de se fusionner avec des exclamations. Autrement dit, l'expression de l'interrogation ne se sépare pas vraiment de l'expression d'une attitude ni d'une émotion dans la communication entre Vladimir, Estragon et Pozzo. L'enseignement du FLE devrait lui aussi miser plus sur la proximité qui existe entre les interrogations et les exclamations, car il s'agit d'une singularité prosodique qui est propre, pas seulement au théâtre de Beckett, mais surtout à la langue française en général. Le texte littéraire le met bien en évidence alors que l'enseignement du FLE devrait insister un peu plus sur l'intonation expressive qui unit les exclamations et les interrogations et qui est un vrai défi pour beaucoup d'apprenants (finnophones) du français.

Effectivement, chaque texte choisi a toujours un certain effet plus ou moins important sur la production orale des apprenants. Si les apprenants finnophones auraient lu, à la place de l'extrait de Beckett, une scène issue d'une pièce de théâtre de Molière, leur production du français langue étrangère aurait sans doute été influée plus ou moins par certaines caractéristiques propres au français parlé du $21^{\mathrm{e}}$ siècle, qu'ils auraient entendues en classe ou en dehors de la classe, au détriment des finesses du français du $17^{\mathrm{e}}$ siècle. Mais le choix du texte ne remet pas en question les résultats, car une partie des erreurs d'intonation sont étroitement liées à d'autres difficultés de prononciation rencontrées à la lecture du texte en question. En effet, dans le feedback que les apprenants nous ont écrit, un des apprenants a constaté ceci: «Stressée, la concentration va à la prononciation des mots et on fait moins attention à l'intonation. Surtout si on oublie le sens de la phrase». Ce qui leur a aussi compliqué la tâche, c'était la coexistence de plusieurs faits phonétiques dont l'apprenant de L2 devait tenir compte en lisant le texte écrit en langue étrangère: "Je ne trouve pas difficile de produire l'intonation (en français), mais combiné avec les règles de prononciation et la parole rapide, c'est difficile», constate à juste titre un apprenant. Cela dit, nous pouvons conclure que pédagogiquement, il serait utile de proposer aux apprenants de FLE des exercices de phonétique qui leur permettrait de pratiquer plusieurs éléments de phonétique du français à la fois, sans isoler les exercices d'intonation des autres exercices de phonétique comme c'est la coutume dans plusieurs manuels de FLE.

Notre étude apporte ainsi des preuves supplémentaires du fait déjà montré par des études antérieures (Horgue, 2010; Jilka, 2007; MacDonald, 2011; Santiago et 
Delais-Roussarie, 2015) qu'en ce qui concerne la prosodie de la parole des locuteurs L2, tous les traits qui diffèrent de ceux typiques des locuteurs natifs ne peuvent pas être expliqués par la transmission directe des traits prosodiques de la L1.

Par la suite, il serait intéressant d'étudier la production et l'interprétation de la prosodie des énoncés interrogatifs et exclamatifs dans la parole des apprenants finnophones du français qui sont issus de l'immigration et dont la L1 n'est pas le finnois ni le suédois mais une autre langue - telle que l'estonien, le russe, l'anglais, le somalien, l'arabe qui sont actuellement les langues les plus parlées en Finlande après les langues nationales. Il serait également intéressant de faire une étude pour comprendre si les apprenants finlandais captent les messages implicites que les locuteurs natifs du français envoient par le biais de la prosodie, car nous sommes d'avis qu'au $21^{\mathrm{e}}$ siècle, l'enseignement des langues étrangères pourrait et devrait être utilisé de façon à améliorer la capacité des jeunes de ressentir, de comprendre et de distinguer diverses attitudes et différents affects devenus de plus en plus complexes, qu'ils soient les leurs ou ceux d'autrui.

\section{REFERENCES}

Albert, M.-Cl. et Souchon, M. (2000). Les Textes littéraires en classe de langue. Paris: Hachette.

Anttila, H. (2008). The effect of interrogative function on intonation in spontaneous and read Finnish. [Mémoire de Master]. Helsinki: Helsingin yliopisto. URL: http://urn.fi/URN:NBN:fi-fe200807301740, consulté le 3 février 2021.

Anttila, H. (2009). Interrogative intonation in spontaneous Finnish. In: V. de Silva and R. Ullakonoja (eds.), Phonetics of Russian and Finnish. General Description of Phonetic Systems. Experimental Studies on Spontaneous and Read-aloud Speech. Frankfurt am Main: Peter Lang, pp. 167-176.

Beckett, S. (1952). En attendant Godot. Paris: Éditions de Minuit.

Beirne, E. (2020). What a feeling! A multistage, multimethod Investigation of emotions and their antecedents in an Irish language MOOC. PhD thesis, Dublin City University. http://doras.dcu.ie/24749/

Boersma, P. et Weenink, D. (2021). Praat: doing phonetics by computer. [Logiciel]. URL: http://www.praat. org/, consulté le 19 février 2021.

Common European Framework of Reference for Languages (CEFR) (2021). Qualitative aspects of spoken language use - Table 3 (CEFR 3.3): Common Reference levels. URL: https:/www.coe.int/en/web/ common-european-framework-reference-languages/table-3-cefr-3.3-common-reference-levels-qualitativeaspects-of-spoken-language-use, consulté le 10 février 2021.

Coveney, A. (2011). L'interrogation directe. Travaux de linguistique, 2011/2 n63: 112-145.

Defays, J.-M., Delbart, A.-R., Hammami S. et Saenen, F. (2014). La littérature en FLE. État des lieux et nouvelles perspectives. Paris: Hachette.

Delais-Roussarie, E., Post, B., Avanzi, M., Buthke, C., Di Cristo, A., Feldhausen, I., Jun, S.-A., Martin, Ph., Meisenburg, T., Rialland, A., Sichel-Bazin, R. et Yoo, H.-Y. (2015). Intonational Phonology of French: Developing a ToBI system for French. In: S. Frota and P. Prieto (Eds.), Intonation in Romance. Oxford: Oxford University Press.

Delais-Roussarie, E. et Herment, S. (2018). Intonation et interprétation des questions : un puzzle pluridimensionnel. In: M.-J. Béguelin, A. Coveney and A. Guryev (Eds.), L'interrogative en français. Bern, Berlin, Bruxelles, New York, Oxford, Warszawa, Wien: Peter Lang, pp. 51-78.

Dewaele, J.-M. (2005). Investigating the Psychological and Emotional Dimensions. Instructed Language Learning: Obstacles and Possibilities. In: The Modern Language Journal. Volume 89. 9/2005. 367-380.

Di Cristo, A. (1998). Intonation in French. In: D. Hirst and A. Di Cristo (Eds.), Intonation Systems: A Survey of Twenty Languages. Cambridge: Cambridge University Press, pp. 195-218.

Lefeuvre, F. (1999). La phrase averbale en français. Paris: L'Harmattan.

Fónagy, I. et Bérard, E. (1973). Questions totales simples et implicatives en français parisien. In Grundstrom, A. \& Léon, P.R. (Eds.) Interrogation et Intonation. (= Studia Phonetica 8): 53-97.

Gardes-Tamine, J. (2004): La grammaire 2. Syntaxe. Paris: Armand Colin. 
Grammaire du français contemporain (1964). Paris: Libraire Larousse.

Granath, M., Laine, K. et Penttilä, R. (2014) Escalier 1. Helsinki: Sanoma Pro Oy.

Gussenhoven, C. (2004). The phonology of tone and intonation. Cambridge: Cambridge University Press.

Hirschsprung N. et Tricot, T. (2017). Cosmopolite 1. Paris: Hachette.

Hirst, D. et Di Cristo, A. (eds.) (1998). Intonation Systems. A Survey of Twenty languages. Cambridge: Cambridge University Press.

Horgues, C. (2010). Prosodie de l'accent français en anglais et perception par des auditeurs anglophones. [Thèse de doctorat non publiée]. Paris: Université Paris Diderot Paris 7.

Iivonen, A. (1978). Is there interrogative intonation in Finnish? In: E. Gårding, G. Bruce and R. Bannert (Eds.), Nordic Prosody. Papers from a Symposium. Lund: Lund University, pp. 43-53.

Iivonen, A. (1998). Intonation in Finnish. In: D. Hirst and A. Di Cristo (Eds.), Intonation Systems: A Survey of Twenty Languages. Cambridge: Cambridge University Press, pp. 311-327.

Iivonen, A. (2009). Finnish sentence accent and intonation. In: V. de Silva and R. Ullakonoja (Eds.), Phonetics of Russian and Finnish. General Description of Phonetic Systems. Experimental Studies on Spontaneous and Read-aloud Speech. Frankfurt am Main: Peter Lang, pp. 67-73.

Iivonen, A., Nevalainen, T., Aulanko, R. et Kaskinen, H. (1987). Puheen intonaatio. Helsinki: Gaudeamus.

Jilka, M. (2007). Different manifestations and perceptions of foreign accent in intonation. In: J. Trouvain and U. Gut (Eds.), Non-native prosody: Phonetic descriptions and teaching practice. Berlin: Mouton de Gruyter, pp. 77-96.

Kivivirta N., Kuikka, T., Mauffret D., Sarimo J. et Virtanen, T. (2015). J'aime 1. Helsinki: Otava.

MacDonald, D. (2011). Second language acquisition of English question intonation by Koreans. In: L. Amstrong (Ed.), Proceedings of the 2011 annual conference of the Canadian Linguistic association. Fredericton: University of New Brunswick. URL: http://homes.chass.utoronto.ca/ cla-acl/actes2011/ MacDonald_2011.pdf, consulté le 2 février 2021.

Mennen, I. (2007). Phonological and phonetic influences in non-native intonation. In: J. Trouvain and U. Gut (Eds.), Non-native prosody: Phonetic descriptions and teaching practice. Berlin: Mouton de Gruyter, pp. 53-76.

Mennen, I. et De Leeuw, E. (2014). Beyond Segments: Prosody in SLA. Studies in Second Language Acquisition, 36 (2): 183-194. URL: https://doi.org/10.1017/S0272263114000138, consulté le 19 février 2021.

Mertens, P. (2008). Syntaxe, prosodie et structure informationnelle : une approche prédictive pour l'analyse de l'intonation dans le discours. Travaux de Linguistique, 56/1: 87-124.

Mertens, P. (2004). The prosogram: Semi-automatic transcription of prosody based on a tonal perception model. In: Proceedings of Speech Prosody 2004: Nara, Japan, March 23-26, 2004. URL: https://iscaspeech.org/archive/sp2004/papers/sp04_549.pdf, consulté le 10 février 2021.

Morange, Séverine (2005). Approches structurale, prosodique, psycho-cognitive de quelques propriétés linguistiques subjectives de la parole : l'exemple de trois Montmartrois âgés. [Thèse de doctorat]. Paris: Université de la Sorbonne Nouvelle (Paris 3).

Morel, M.-A. et Danon-Boileau, L. (1998). Grammaire de l'intonation. L'Exemple du français oral. Paris / Gap: Ophrys.

Pekrun, R. et Linnenbrink-Garcia, L. (eds.) (2014). Educational psychology handbook series. International handbook of emotions in education. New York: Routledge/Taylor \& Francis Group.

Rasier, L. et Hiligsmann, P. (2007). Prosodic transfer from L1 to L2. Theoretical and methodological issues. Nouveaux cahiers de linguistique française, 28: 41-66.

Riegel, M., Pellat, J.-C. et Rioul, R. (1994). Grammaire méthodique du français. Paris : PUF.

Rossi, M. (1999). L'intonation, le système du français : description et modélisation. Paris: Ophrys.

Santiago, F. et Delais-Roussarie, E. (2015). The acquisition of Question Intonation by Mexican Spanish Learners of French. In: E. Delais-Roussarie, M. Avanzi and S. Herment (Eds.), Prosody and Language in Contact: L2 Acquisition, Attrition and Languages in Multilingual Situations. New-York/Heldelberg: Springer-Verlag, pp. 243-270.

Tyni, M.-R. (2021). Enseignement de l'interrogation directe dans les manuels de FLE J'aime et Escalier. [Mémoire de master]. Helsinki: Université de Helsinki. 


\section{ANNEXE 1}

TEXTE LU PAR LES INFORMATEURS : EN ATTENDANT GODOT (Beckett, 1952: 118-119)

Pozzo. - A moi !

Estragon. - C’est toute l'humanité. (Silence.) Regarde-moi ce petit nuage.

Vladimir (levant les yeux). - Où ?

Estragon. - Là, au zénith.

Vladimir. - Eh bien ? (Un temps.) Qu'est-ce qu'il a de si extraordinaire?

Silence.

Estragon. - Passons maintenant à autre chose, veux-tu ?

Vladimir. - J'allais justement te le proposer.

Estragon. - Mais à quoi ?

Vladimir. - Ah, voilà !

Silence.

Estragon. - Si on se levait, pour commencer ?

Vladimir. - Essayons toujours.

Ils se lèvent.

Estragon. - Pas plus difficile que ça.

Vladimir. - Vouloir, tout est là.

Estragon. - Et maintenant ?

Pozzo. - Au secours!

Estragon. - Allons-nous-en.

Vladimir. - On ne peut pas.

Estragon. - Pourquoi ?

Vladimir. - On attend Godot.

Estragon. - C'est vrai. (Un temps.) Que faire?

Pozzo. - Au secours!

Vladimir. - Si on le secourait ?

Estragon. - Qu'est-ce qu'il faut faire?

Vladimir. - Il veut se lever.

Estragon. - Et après ?

Vladimir. - Il veut qu'on l'aide à se lever.

Estragon. - Eh bien, aidons-le. Qu'est-ce qu'on attend ?

Ils aident Pozzo à se lever, s'écartent de lui. Il retombe.

Vladimir. - Il faut le soutenir (Même jeu. Pozzo reste debout entre les deux, pendu à leur cou.) Il faut qu'il se réhabitue à la station debout. (A Pozzo.) Ça va mieux ?

Pozzo. - Qui êtes-vous?

Vladimir. - Vous ne nous remettez pas?

Pozzo. - Je suis aveugle.

Silence.

Estragon. - Peut-être qu'il voit clair dans l'avenir?

Vladimir (à Pozzo). - Depuis quand ?

Pozzo. - J'avais une très bonne vue - mais êtes-vous des amis ?

Estragon (riant bruyamment). - Il demande si nous sommes des amis !

Vladimir. - Non, il veut dire des amis à lui.

Estragon. - Et alors?

Vladimir. - La preuve, c'est que nous l'avons aidé. 
ANNEXE 2

TABLEAUX PRÉSENTANT LES VALEURS DE L'ÉTENDUE DES MOUVEMENTS DE LA FO À LA FIN DES QUESTIONS ÉTUDIÉES (DT) AINSI QUE LES ÉCARTS-TYPES (DT) ET LES MOYENNES (Hz) DE LA FO DES INFORMATEURS

Tableau 3. Les valeurs de l'étendue des mouvements de la $f_{0}$ à la fin des questions étudiées. Les écarts-types et les moyennes de la $f_{0}$ des locuteurs. Apprenants finnophones (de 1 à 8 )

\begin{tabular}{|c|c|c|c|c|c|c|c|c|}
\hline \multirow[b]{2}{*}{ Question: } & \multicolumn{8}{|c|}{$\begin{array}{l}\text { Valeurs de l'étendue des mouvements de la } f_{o} \text { à la fin des } \\
\text { questions étudiées (dt), écarts-types (dt) et moyennes }(\mathrm{Hz}) \\
\text { de la } f_{o} \text { des locuteurs. } \\
\text { Apprenants finnophones (de } 1 \text { à } 8 \text { ). } \mathrm{F} \text { : voix féminine. M : voix mascu- } \\
\text { line. }\end{array}$} \\
\hline & 1FIN_F & 2FIN_F & 3FIN_F & 4FIN_F & 5FIN_F & 6FIN_M & 7FIN_F & 8FIN_F \\
\hline $\begin{array}{l}\text { "Passons maintenant à } \\
\text { autre chose, veux-tu ?» }\end{array}$ & $+5,7$ & $+4,7$ & $+4,3$ & $-1,7$ & $+6,6$ & $-17,0$ & $+4,1$ & $+9,8$ \\
\hline "Ça va mieux?» & $+11,9$ & $+8,5$ & $+6,5$ & $+6,1$ & $+7,0$ & $-2,2$ & $+8,3$ & $+12,9$ \\
\hline $\begin{array}{l}\text { "Qu'est-ce qu'il y a de } \\
\text { si extraordinaire?» }\end{array}$ & $+7,4$ & $-12,6$ & $+6,1$ & $-0,4$ & $-0,4$ & $-5,5$ & $-3,5$ & $+8,8$ \\
\hline «Pourquoi? ? & $+6,5$ & $+6,0$ & $+6,7$ & $+2,2$ & $+6,0$ & $+3,3$ & $+2,1$ & $+11,6$ \\
\hline "Qui êtes-vous?» & $+9,8$ & $+9,0$ & $+7,8$ & $+3,2$ & $-1,1$ & $-1,7$ & $-3,3$ & $-9,9$ \\
\hline Moyenne de la $f_{O}$ & 192,6 & 219,6 & 206,1 & 287,2 & 216,5 & 136,8 & 202,8 & 240,1 \\
\hline Écart-type de la $f_{0}$ & 4,1 & 3,5 & 4,5 & 3,2 & 4,4 & 3,0 & 4,5 & 4,4 \\
\hline
\end{tabular}

Tableau 4. Les valeurs de l'étendue des mouvements de la $f_{0}$ à la fin des questions étudiées. Les écartstypes et les moyennes de la $f_{0}$ des locuteurs. Apprenants finnophones (de 9 à 15)

\begin{tabular}{|c|c|c|c|c|c|c|c|}
\hline \multirow[b]{2}{*}{ Question: } & \multicolumn{7}{|c|}{$\begin{array}{l}\text { Valeurs de l'étendue des mouvements de la } f_{o} \text { à la fin des } \\
\text { questions étudiées (dt), écarts-types (dt) et moyennes }(\mathrm{Hz}) \text { de la } f_{o} \\
\text { des locuteurs. Apprenants finnophones (de } 9 \text { à 15). F : voix féminine. } \\
\text { M: voix masculine. }\end{array}$} \\
\hline & 9FIN_F & 10FIN_F & $11 F I N \_M$ & 12FIN_F & 13FIN_M & 14FIN_F & $15 F I N \_N$ \\
\hline $\begin{array}{l}\text { «Passons maintenant } \\
\text { à autre chose, veux-tu } \\
\text { ?» }\end{array}$ & $+6,8$ & $+4,8$ & $+9,6$ & $+5,6$ & $+2,1$ & $+3,9$ & $+3,2$ \\
\hline «Ça va mieux?» & $+12,1$ & $+11,2$ & $+11,4$ & $+15,6$ & $+3,7$ & $+7,1$ & $+7,2$ \\
\hline $\begin{array}{l}\text { "Qu'est-ce qu'il y a de } \\
\text { si extraordinaire?» }\end{array}$ & $+11,5$ & $-13,5$ & $+10,4$ & $+7,7$ & $+5,4$ & $+4,6$ & $-2,6$ \\
\hline «Pourquoi? » & $+5,6$ & $+5,5$ & $+8,1$ & $+9,0$ & $+1,5$ & $+3,7$ & $-7,7$ \\
\hline "Qui êtes-vous?» & $+18,4$ & $+10,9$ & $+14,5$ & $+9,3$ & $+3,7$ & $-7,0$ & $-5,8$ \\
\hline Moyenne de la $f_{0}$ & 222,6 & 235,3 & 156,2 & 236,2 & 137,0 & 210,8 & 244,4 \\
\hline Écart-type de la $f_{o}$ & 5,8 & 4,3 & 4,0 & 3,5 & 2,6 & 3,1 & 4,0 \\
\hline
\end{tabular}


Tableau 5. Les valeurs de l'étendue des mouvements de la $f_{0}$ à la fin des questions étudiées. Les écartstypes et les moyennes de la $f_{0}$ des locuteurs. Locuteurs natifs

\begin{tabular}{|c|c|c|c|c|c|}
\hline \multirow[b]{2}{*}{ Question: } & \multicolumn{5}{|c|}{$\begin{array}{l}\text { Valeurs de l'étendue des mouvements de la } \\
f_{o} \text { à la fin des questions étudiées (dt), } \\
\text { écarts-types (dt) et moyennes (Hz) de la } f_{o} \\
\text { des locuteurs. Locuteurs natifs. } F \text { : voix } \\
\text { féminine. M : voix masculine. }\end{array}$} \\
\hline & 1FR_F & 2FR_F & 3FR_F & 4FR_F & 5FR_F \\
\hline «Passons maintenant à autre chose, veux-tu ?» & $+4,5$ & $+4,7$ & $+9,5$ & $+7,2$ & $+3,5$ \\
\hline «Ça va mieux?» & $+12,1$ & $+12,4$ & $+9,2$ & $+8,4$ & $+10,2$ \\
\hline "Qu'est-ce qu'il y a de si extraordinaire?» & $+1,8$ & $-4,5$ & $+3,2$ & $+1,9$ & $+0,1$ \\
\hline «Pourquoi? » & $+0,1$ & $-10,1$ & $+3,7$ & $+1,9$ & $+3,4$ \\
\hline "Qui êtes-vous?» & $-8,0$ & $-3,8$ & $+6,8$ & $-5,1$ & $-3,0$ \\
\hline Moyenne de la $f_{O}$ & 233,1 & 209,4 & 241,1 & 222,1 & 211,3 \\
\hline Écart-type de la $f_{0}$ & 4,2 & 3,6 & 3,6 & 3,8 & 3,3 \\
\hline
\end{tabular}

Cite this article: Wiklund M and Riippa A (2022). L'intonation des énoncés interrogatifs dans la parole des apprenants finnophones du français. Journal of French Language Studies 32, 383-411. https://doi.org/10. $1017 /$ S0959269521000211 